\title{
Complete off-shell effects in top quark pair hadroproduction with leptonic decay at next-to-leading order
}

\author{
Giuseppe Bevilacqua, ${ }^{a, b}$ Michat Czakon, ${ }^{b}$ Andreas van Hameren, ${ }^{c}$ \\ Costas G. Papadopoulos ${ }^{a}$ and Matgorzata Worek ${ }^{d}$ \\ ${ }^{a}$ Institute of Nuclear Physics, NCSR Demokritos, \\ GR-15310 Athens, Greece \\ ${ }^{b}$ Institut für Theoretische Teilchenphysik und Kosmologie, \\ RWTH Aachen University, D-52056 Aachen, Germany \\ ${ }^{c}$ The H. Niewodniczański Institute of Nuclear Physics, \\ Polisch Academy of Sciences, Radzikowskiego 152, PL-31342 Cracow, Poland \\ ${ }^{d}$ Fachbereich C Physik, Bergische Universität Wuppertal, \\ D-42097, Wuppertal, Germany \\ E-mail: bevilacqua@physik.rwth-aachen.de, \\ mczakon@physik.rwth-aachen.de, Andre.Hameren@ifj.edu.pl, \\ Costas.Papadopoulos@cern.ch, worek@physik.uni-wuppertal.de
}

ABstRACT: Results for next-to-leading order QCD corrections to the $p p(p \bar{p}) \rightarrow t \bar{t} \rightarrow$ $W^{+} W^{-} b \bar{b} \rightarrow e^{+} \nu_{e} \mu^{-} \bar{\nu}_{\mu} b \bar{b}+X$ processes with complete off-shell effects are presented for the first time. Double-, single- and non-resonant top contributions of the order $\mathcal{O}\left(\alpha_{s}^{3} \alpha^{4}\right)$ are consistently taken into account, which requires the introduction of a complex-mass scheme for unstable top quarks. Moreover, the intermediate $W$ bosons are treated off-shell. Comparison to the narrow width approximation for top quarks, where non-factorizable corrections are not accounted for is performed. Besides the total cross section and its scale dependence, several differential distributions at the TeVatron run II and the LHC are given. In case of the TeVatron the forward-backward asymmetry of the top is recalculated afresh. With inclusive selection cuts, the forward-backward asymmetry amounts to $A_{F B}^{t}=$ $0.051 \pm 0.0013$. Furthermore, the corrections with respect to leading order are positive and of the order $2.3 \%$ for the TeVatron and $47 \%$ for the LHC. A study of the scale dependence of our NLO predictions indicates that the residual theoretical uncertainty due to higher order corrections is $8 \%$ for the TeVatron and $9 \%$ for the LHC.

KeYwords: Jets, NLO Computations, Hadronic Colliders, QCD 


\section{Contents}

1 Introduction 1

2 Theoretical framework $\quad 2$

2.1 Born level 2

2.2 The virtual corrections 4

2.3 The real emission $\quad 5$

$\begin{array}{llr}2.4 & \text { Phase space generation } & 6\end{array}$

3 Numerical results $\quad 7$

3.1 Setup 7

3.2 Results for the TeVatron run II 9

$\begin{array}{lll}3.3 & \text { Results for the LHC } & 17\end{array}$

4 Conclusions $\quad 22$

\section{Introduction}

The $t \bar{t}$ production process is a copious source of $W$-pairs and, hence, of isolated leptons at the TeVatron and the LHC. In consequence it is intensely studied as a signal at these colliders. In view of the large production rate, precise and direct measurements are possible, which require a detailed theoretical understanding. In addition, it constitutes an important background for many new particle searches. Examples include the leptonic signals for cascade decays of supersymmetric particles or searches for $H \rightarrow W^{+} W^{-}$and $H \rightarrow \tau^{+} \tau^{-}$decays.

Even though, the first results for next-to-leading order (NLO) QCD corrections to heavy quark production were presented more than twenty years ago [1-4], recent progress in NLO [5-9] and next-to-next-to leading order (NNLO) [10-15] calculations, as well as next-to-next-to-leading-log resummations (NNLL) [16-22] for inclusive $t \bar{t}$ hadroproduction is truly astonishing.

The list for the more exclusive channels is just as impressive: NLO QCD corrections have been calculated for the $t \bar{t} H$ signal [23-28], where the Higgs boson has been treated as a stable particle. Most recently the factorizable QCD corrections to this process have been presented [29], where higher order corrections to both production and decay of the Higgs boson to a $b \bar{b}$ pair have been calculated with the latter modeled by the Higgs propagator with a fixed width. Moreover, NLO QCD corrections to a variety of $2 \rightarrow 3$ backgrounds processes $t \bar{t} j$ [30-32], $t \bar{t} Z$ [33] and $t \bar{t} \gamma$ [34] have been obtained. Most recently, NLO QCD corrections to $2 \rightarrow 4$ backgrounds $t \bar{t} b \bar{b}$ [35-38] and $t \bar{t} j j$ [39] have also been evaluated. 
Usually, $t \bar{t}$ production is restricted to on-shell states and decays if available are treated in the narrow-width approximation (NWA), which effectively decouples top production and decay. The NWA allows to neglect non-resonant as well as non-factorizable amplitude contributions, thus leading to significant simplifications for calculations of higher order corrections. Whenever resonant top production dominates, as it does for very inclusive cuts, this approximation is of course well motivated. In some cases calculations have been further simplified by also treating the decaying $W$ bosons as on-shell particles.

Naturally, the accuracy of these approximations needs to be tested, which requires a full calculation of off-shell effects. One thus needs a calculation which includes both resonant and non-resonant contributions, using finite width top-quark propagators, which correctly includes interference effects between the various contributions. The purpose of this paper is to present such a complete calculation for $t \bar{t}$ production at NLO QCD level. In addition to merging resonant and non-resonant effects for the top quarks, we also include finite width effects for the $W$ bosons, i.e. we consider NLO QCD corrections to the general $e^{+} \nu_{e} \mu^{-} \bar{\nu}_{\mu} b \bar{b}$ final state.

In addition, all selection strategies based on next-to-leading order simulations, which have been devised for the efficient suppression of $t \bar{t}$ background, are at present optimized against top production in the NWA. Within our approach, presented in the form of a flexible Monte Carlo program which allows to study NLO QCD corrections to cross sections and kinematic distributions with arbitrary cuts on particles in the final state and with full spin correlations, it is possible to reexamine the quality of the chosen selection with improved accuracy.

The paper is organized as follows. In section 2 we briefly describe the calculation of the NLO corrections. Numerical results for the integrated and differential cross sections are presented in section 3 both for the TeVatron and the LHC. Finally, we conclude in section 4 .

\section{Theoretical framework}

\subsection{Born level}

At Born level the partonic reactions are

$$
\begin{aligned}
g g & \rightarrow e^{+} \nu_{e} \mu^{-} \bar{\nu}_{\mu} b \bar{b} \\
q \bar{q} & \rightarrow e^{+} \nu_{e} \mu^{-} \bar{\nu}_{\mu} b \bar{b}
\end{aligned}
$$

where $q$ stands for up- or down-type quarks. The $\mathcal{O}\left(\alpha_{s}^{2} \alpha^{4}\right)$ contributions to the $e^{+} \nu_{e} \mu^{-} \bar{\nu}_{\mu} b \bar{b}$ process can be subdivided into three classes, namely diagrams containing two top quark propagators that can become resonant, diagrams containing only one top quark resonance and finally diagrams without any top quark resonance. Regarding the $W^{ \pm}$resonances one can distinguish only two subclasses, double- and single-resonant gauge boson contributions. A few examples of Feynman diagrams contributing to the leading order $g g \rightarrow e^{+} \nu_{e} \mu^{-} \bar{\nu}_{\mu} b \bar{b}$ subprocess are presented in figure 1.

Since the produced top quarks are unstable particles, the inclusion of the decays is performed in the complex mass scheme, which for LO is described in ref. [40, 41]. It fully 

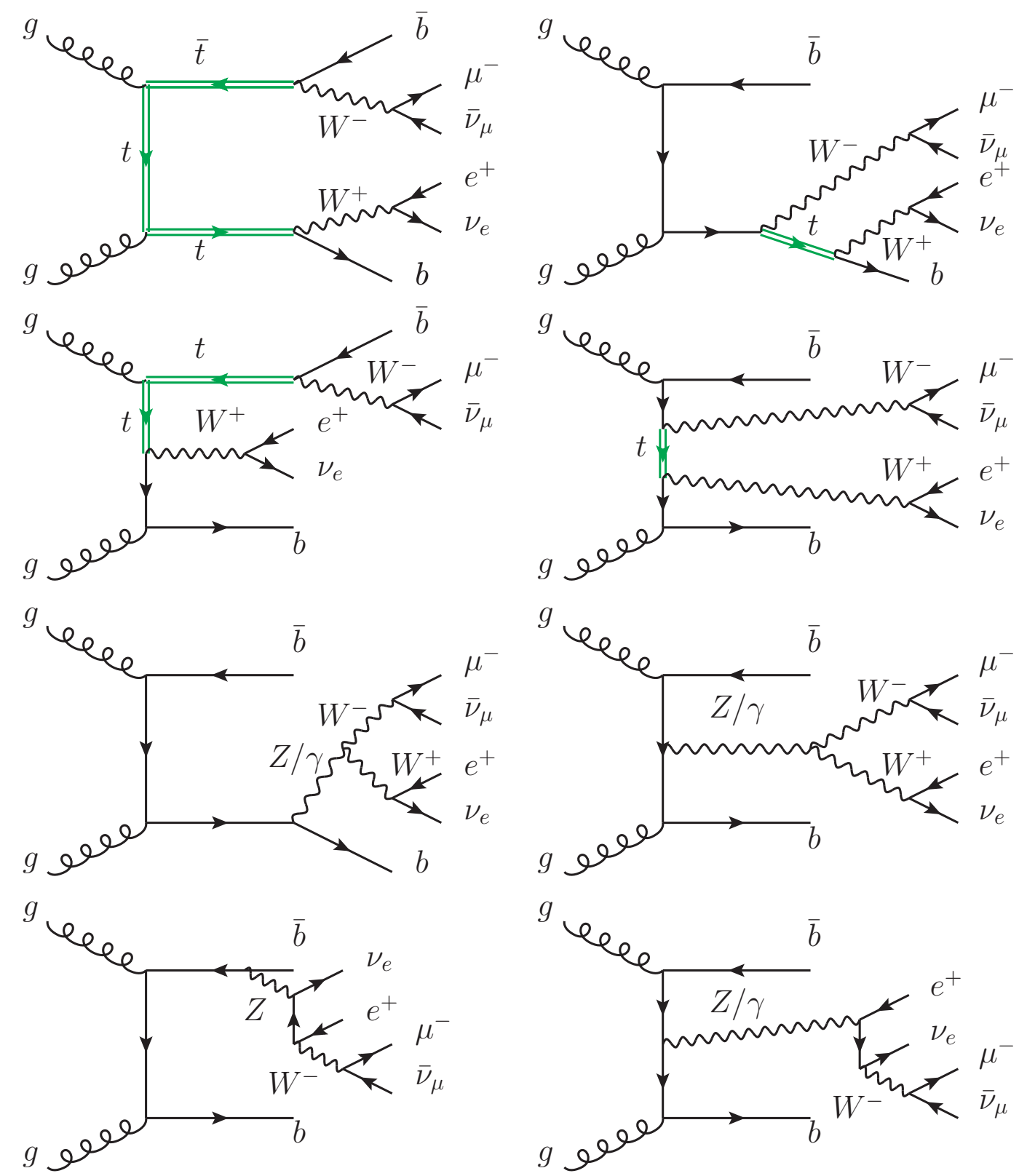

Figure 1. Representative Feynman diagrams contributing to the leading order process $g g \rightarrow$ $e^{+} \nu_{e} \mu^{-} \bar{\nu}_{\mu} b \bar{b}$ at $\mathcal{O}\left(\alpha_{s}^{2} \alpha^{4}\right)$, with different off-shell intermediate states: double-, single-, and nonresonant top quark contributions.

respects gauge invariance and is straightforward to apply. In the amplitude (at LO and NLO) we simply perform the substitution

$$
\left(\not p-m_{t}+i \epsilon\right)^{-1} \rightarrow\left(\not p-\mu_{t}+i \epsilon\right)^{-1}, \quad \quad \mu_{t}^{2}=m_{t}^{2}-i m_{t} \Gamma_{t} .
$$

Since we are interested in NLO QCD corrections, gauge bosons are treated within the fixed width scheme. Our LO results have been generated with the Helac-Dipoles [42] package and cross checked with Helac-Phegas [43, 44], a generator for all parton level processes 
in the Standard Model, which has, on its own, already been extensively used and tested in phenomenological studies see e.g. [45-49]. The integration over the fractions $x_{1}$ and $x_{2}$ of the initial partons is optimized with the help of PARNI [50]. The phase space integration is executed with the help of KALEU [51] and cross checked with PHEGas [52], both general purpose multi-channel phase space generators.

Furthermore, results have been checked against another program that computes the $t \bar{t}$ production cross section with top decays, namely MCFM [53]. A perfect agreement has been found with our results, both for the TeVatron and the LHC, once top quarks and $W$ gauge bosons have been put on shell in the HeLAC-DiPOLES package. We additionally reproduced results presented in ref. [8] again assuming that both tops and $W$ 's are on shell.

\subsection{The virtual corrections}

The virtual corrections consist of the 1-loop corrections to the LO reactions. One can classify the corrections into self-energy, vertex, box-type, pentagon-type and hexagon-type corrections. Typical examples of the virtual graphs are shown in figure 2. In evaluating the virtual corrections, the HELAC-1Loop [54] approach is used. It is based on the Helac-Phegas program to calculate all tree-order like ingredients and the OPP [55] reduction method. The cut-constructible part of the virtual amplitudes is computed using the CutTools [56] code. The rational term $R_{1}$ of the amplitude is computed by the CuTTools code as well, whereas the $R_{2}$ term, by the use of extra Feynman rules as described in $[56,57]$. Numerical results are obtained using the same methods as described in [37].

As explained before, the process under consideration requires a special treatment of unstable top quarks, which is achieved within the complex-mass scheme [40]. At the one-loop level the appearance of a non-zero top-quark width in the propagator requires the evaluation of scalar integrals with complex masses, for which the program ONELOOP $[54,58]$ is used. We also need mass renormalization for the top quark, which, for consistency, is done by using a complex mass in the well known on-shell mass counterterm. The preservation of gauge symmetries (Ward Identities) [40, 59-61] by this approach has been explicitly checked up to the one-loop level.

Although finite width effects have been studied routinely at tree order, the same is not true for calculations at the one loop level. A novel aspect of the introduction of a nonzero width is the effect on the infrared structure of the scattering amplitudes. Working in dimensional regularization, soft and collinear singularities arise. When massive particles acquire a complex mass, the soft $1 / \epsilon$-singularities due to the exchange of gluons, are replaced by factors proportional to $\log \left(\Gamma_{t} / m_{t}\right)$, that become singular in the limit $\Gamma_{t} \rightarrow 0$. We have explicitly checked that including all contributions, factorizable and non-factorizable, the usual cancellation of infrared $1 / \epsilon^{2}$ and $1 / \epsilon$ poles between virtual and real corrections, the latter represented by the $\mathcal{I}(\epsilon)$-operator, takes place. This means that a partial cancellation of $\log \left(\Gamma_{t} / m_{t}\right)$ terms happens within the virtual corrections alone. Nevertheless logarithmic enhancements remain in the finite part of the virtual corrections and have to be cancelled by corresponding terms from the real corrections, since they represent the same soft singularities, dimensionally regularized in the case of on-shell particles. 

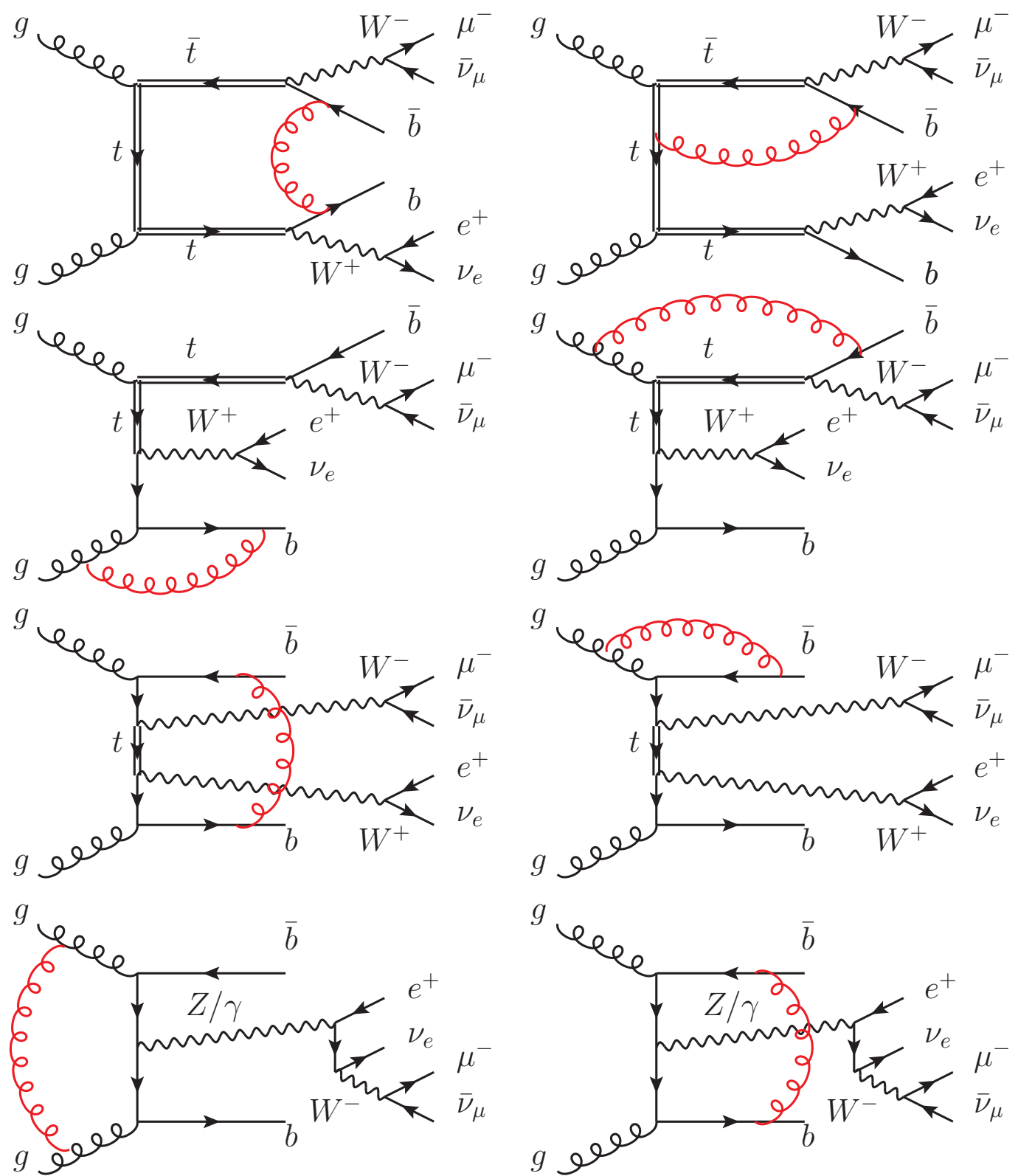

Figure 2. Representative Feynman diagrams contributing to the virtual corrections to the partonic subprocess $g g \rightarrow e^{+} \nu_{e} \mu^{-} \bar{\nu}_{\mu} b \bar{b}$ at $\mathcal{O}\left(\alpha_{s}^{3} \alpha^{4}\right)$.

\subsection{The real emission}

The generic processes for the real corrections are given by

$$
\begin{aligned}
g g & \rightarrow e^{+} \nu_{e} \mu^{-} \bar{\nu}_{\mu} b \bar{b} g \\
q g & \rightarrow e^{+} \nu_{e} \mu^{-} \bar{\nu}_{\mu} b \bar{b} q \\
g q & \rightarrow e^{+} \nu_{e} \mu^{-} \bar{\nu}_{\mu} b \bar{b} q \\
q \bar{q} & \rightarrow e^{+} \nu_{e} \mu^{-} \bar{\nu}_{\mu} b \bar{b} g
\end{aligned}
$$


(where again $q$ stands for up- or down-type quarks) and include all possible contributions of the order of $\mathcal{O}\left(\alpha_{s}^{3} \alpha^{4}\right)$. The complex mass scheme for unstable top quarks has been implemented in complete analogy to the LO case.

We employ the dipole subtraction formalism [62] to extract the soft and collinear infrared singularities and to combine them with the virtual corrections. Specifically, the formulation [63] for massive quarks has been used with the extension to arbitrary helicity eigenstates of the external partons [42], as implemented in HelaC-Dipoles. In the case at hand, the number of dipoles is as follows: 27 for the process $g g \rightarrow e^{+} \nu_{e} \mu^{-} \bar{\nu}_{\mu} b \bar{b} g$ and 15 for processes $q \bar{q} \rightarrow e^{+} \nu_{e} \mu^{-} \bar{\nu}_{\mu} b \bar{b} g, q g \rightarrow e^{+} \nu_{e} \mu^{-} \bar{\nu}_{\mu} b \bar{b} q$ and $g q \rightarrow e^{+} \nu_{e} \mu^{-} \bar{\nu}_{\mu} b \bar{b} q$. Let us stress at this point, that, similarly to most authors, we do not use finite dipoles regularizing the quasi-collinear divergence induced by both top quarks moving in the same direction, even though they are implemented in the software. Due to the large top quark mass, they do not improve numerical stability.

Besides the cancellation of divergences, which we have mentioned in the previous section, we have also explored the independence of the results on the unphysical cutoff in the dipole subtraction phase space (see [42] and references therein for details) to further check our calculation.

\subsection{Phase space generation}

In LO calculations, the jet definition consists of a set of phase space cuts not allowing any parton to become arbitrarily soft, and no pair of partons to become arbitrarily collinear. This changes for the real-radiation contribution in NLO calculations, for which single partons are allowed to become arbitrarily soft and single pairs of partons are allowed to become arbitrarily collinear. This means that phase space generators like PHEGAS [52] and KALEU [51], which construct momentum configurations from kinematical variables generated following a priori defined probability densities, cannot be directly applied in their LO set-up, since these densities anticipate the singular behavior of the squared amplitudes, and are typically not defined in the soft and collinear limits. Furthermore, the subtraction terms in the dipole-subtraction scheme, used to eliminate the singularities in the real-radiation phase space integral, do not exactly follow the same peak structure as the tree-level $n+1$-particle matrix element squared, whereas Phegas and Kaleu are designed only to efficiently deal with the latter. We chose to deal with this situation via a multi-channel approach [64], in which a separate channel is associated with each term in the real-subtracted integral, i.e., with the tree-level $n+1$-particle matrix element squared as well as each dipole term.

The channel for the $n+1$-particle matrix element squared generates momenta using an instance of KALEU anticipating the peak structure of this integrand. The phase space defined by promoting the LO cuts to $n+1$ partons is filled in the usual LO approach. The soft and collinear regions "below the cuts" are filled by replacing the densities for the invariants by densities that are integrable in these regions.

All dipole channels also carry their own instances of KALEU, but each of these generates $n$-momentum configurations anticipating the peak structure of the $n$-particle matrix element squared of the underlying process of the dipole term. Such a $n$-momentum config- 
uration is then turned into an $n+1$-momentum configuration by essentially applying the inverse of the phase space mapping performed in the calculation of the dipole contribution itself. This generation of an extra momentum follows exactly the formulas for the parton showers based on the dipole formalism presented in [65] and [66]. The azimuthal angle needed for the construction of the extra momentum is generated with a flat distribution, and the other two variables, traditionally denoted $\left(y_{i j, k}, z_{i}\right)$ for final-final, $\left(x_{i j, a}, z_{i}\right)$ for final-initial, $\left(x_{i j, a}, u_{i}\right)$ for initial-final, and $\left(x_{i, a b}, v_{i}\right)$ for initial-initial dipoles, are generated following self-adaptive densities. This happens "on the fly" during the Monte Carlo integration, following the approach presented in [50]. Finally, each instance of KALEU carries a multi-channel weight in the "highest level" multi-channel density which is optimized during the Monte Carlo integration, and each instance performs its own internal multi-channel optimization, as described in [51].

We have performed a few tests to check the performance of this new approach in case of the $q \bar{q} \rightarrow e^{+} \nu_{e} \mu^{-} \bar{\nu}_{\mu} b \bar{b} g$ subprocess. More precisely, we have made a comparison between three options, namely KALEU with dipole channels, KALEU without dipole channels and Phegas, which does not have dipole channels. Since the computational cost comes mainly from the accepted events, comparisons are made at equal numbers of accepted events. Our findings can be summarized as follows. For the dipole phase space cut-off parameter $\alpha_{\max }=1$, when all dipoles are calculated for each phase space point, Phegas and KaleU without dipoles channels are comparable in terms of errors. KALEU with dipole channels, however, gives an error which is 5 times smaller. Realize that this implies a reduction in the number of events by a factor of 25 to reach the same error. For $\alpha_{\max }=0.01$, when much less dipole subtraction terms are needed per event, the improvement is not so dramatic, and the introduction of the dipole channels reduces the error by a factor 3 compared to KALEU without dipole channels, and a factor 2 compared to PHEGAS, implying a reduction in necessary events by a factor of 9 and 4 respectively.

We conclude that the dipole channels structurally improve the convergence of the phase space integrals for the real-subtracted contribution. It is, however, difficult to express the improvement quantitatively because it depends on the process and the value of parameters like $\alpha_{\max }$.

\section{$3 \quad$ Numerical results}

\subsection{Setup}

We consider the process $p p(p \bar{p}) \rightarrow t \bar{t}+X \rightarrow W^{+} W^{-} b \bar{b}+X \rightarrow e^{+} \nu_{e} \mu^{-} \nu_{\mu} b \bar{b}+X$ both at the TeVatron run II and the LHC i.e. at a center-of-mass energy of $\sqrt{s}=1.96 \mathrm{TeV}$ and $\sqrt{s}=7 \mathrm{TeV}$ correspondingly. For the LHC case we additionally calculate the integrated cross section at a center-of-mass energy $\sqrt{s}=10 \mathrm{TeV}$. We only simulate decays of the weak bosons to different lepton generations to avoid virtual photon singularities stemming from quasi-collinear $\gamma^{*} \rightarrow \ell^{+} \ell^{-}$decays. These interference effects are at the per-mille level for inclusive cuts, as checked by an explicit leading order calculation. The complete $\ell_{1}^{ \pm} \ell_{2}^{\mp}$ cross section (with $\ell_{1,2}=e, \mu$ ) can be obtained by multiplying the result with a leptonflavor factor of 4 . We keep the Cabibbo-Kobayashi-Maskawa mixing matrix diagonal. The 
unstable (anti)top quark is treated within the (gauge invariant) complex-mass scheme, as explained in the previous section. The Standard Model parameters are given the following values within the $G_{\mu}$ scheme [67]:

$$
\begin{array}{cc}
m_{W}=80.398 \mathrm{GeV}, & \Gamma_{W}=2.141 \mathrm{GeV} \\
m_{Z}=91.1876 \mathrm{GeV}, & \Gamma_{Z}=2.4952 \mathrm{GeV}, \\
G_{\mu}=1.16639 \times 10^{-5} \mathrm{GeV}^{-2} \\
\sin ^{2} \theta_{W}=1-m_{W}^{2} / m_{Z}^{2}
\end{array}
$$

The electromagnetic coupling is derived from the Fermi constant $G_{\mu}$ according to

$$
\alpha=\frac{\sqrt{2} G_{\mu} m_{W}^{2} \sin ^{2} \theta_{W}}{\pi} .
$$

For the top quark mass we take $m_{t}=172.6 \mathrm{GeV}$ and all other QCD partons including $b$ quarks as well as leptons are treated as massless. The contribution from the Higgs boson can be neglected since for inclusive cuts it is below 1\%. In our case, however, the $b$-quarks are massless and the Higgs contribution simply vanishes. The top quark width calculated from $[68,69]$ is $\Gamma_{t}^{L O}=1.48 \mathrm{GeV}$ at $\mathrm{LO}$ and $\Gamma_{t}^{N L O}=1.35 \mathrm{GeV}$ at NLO where $\alpha_{s}=\alpha_{s}\left(m_{t}\right)=0.107639510785815$. Mass renormalization is performed in the on-shell scheme. All final-state $b$ quarks and gluons with pseudorapidity $|\eta|<5$ are recombined into jets with separation $\sqrt{\Delta \phi^{2}+\Delta y^{2}}>D=0.4$ in the rapidity-azimuthal angle plane via the following IR-safe algorithmes: the $k_{T}$ algorithm [70-72], the anti- $k_{T}$ algorithm [73] and the inclusive Cambridge/Aachen algorithm (C/A) [74]. The distance measure $d_{i j}$ for these algorithms is defined as

$$
\begin{aligned}
d_{i j} & =\min \left(p_{T}^{2 p}(i), p_{T}^{2 p}(j)\right) \frac{\Delta R_{i j}^{2}}{D^{2}} \\
d_{i B} & =p_{T}^{2 p}(i)
\end{aligned}
$$

where $\Delta R_{i j}=\sqrt{\Delta \phi_{i j}^{2}+\Delta y_{i j}^{2}}$ and the parameter $p$ is equal to 1 for the $k_{T}$ algorithm, 0 for $C / A$ and -1 for anti- $k_{T}$ algorithm. Moreover, we impose the following additional cuts on the transverse momenta and the rapidity of two recombined $b$-jets: $p_{T_{b}}>20 \mathrm{GeV}$, $\left|y_{b}\right|<4.5$ where

$$
p_{T}=\sqrt{p_{x}^{2}+p_{y}^{2}}, \quad y=\frac{1}{2} \ln \left(\frac{E+p_{z}}{E-p_{z}}\right) .
$$

Basic selection is applied to (anti)top decay products to ensure that the leptons are observed inside the detector and are well separated from each other: $p_{T_{\ell}}>20 \mathrm{GeV},\left|\eta_{\ell}\right|<2.5$, $\Delta R_{j \ell}>0.4$, where $j=b, \bar{b}$, and $p_{T_{\text {miss }}}>30 \mathrm{GeV}$. In the following we consistently use the CTEQ6 set of parton distribution functions (PDFs) [75, 76]. More precisely, we take CTEQ6L1 PDFs with a 1-loop running $\alpha_{s}$ in LO and CTEQ6M PDFs with a 2-loop running $\alpha_{s}$ in NLO. The contribution from $b$ quarks in the initial state is neglected, since at LO for inclusive cuts this contribution is suppressed to the per-mille level. The number of active flavors is $N_{F}=5$, and the respective QCD parameters are $\Lambda_{5}^{L O}=165 \mathrm{MeV}$ and 


\begin{tabular}{|c|c|c|c|}
\hline \multirow{2}{*}{ Algorithm } & $\sigma_{\mathrm{LO}}[\mathrm{fb}]$ & $\sigma_{\mathrm{NLO}}^{\alpha_{\max }=1}[\mathrm{fb}]$ & $\sigma_{\mathrm{NLO}}^{\alpha_{\max }=0.01}[\mathrm{fb}]$ \\
\hline${\text { anti- } k_{T}}$ & $34.922 \pm 0.014$ & $35.705 \pm 0.047$ & $35.697 \pm 0.049$ \\
\hline$k_{T}$ & $34.922 \pm 0.014$ & $35.727 \pm 0.047$ & $35.723 \pm 0.049$ \\
\hline $\mathrm{C} / \mathrm{A}$ & $34.922 \pm 0.014$ & $35.724 \pm 0.047$ & $35.746 \pm 0.050$ \\
\hline
\end{tabular}

Table 1. Integrated cross section at LO and NLO for $p \bar{p} \rightarrow e^{+} \nu_{e} \mu^{-} \bar{\nu}_{\mu} b \bar{b}+X$ production at the TeVatron run II with $\sqrt{s}=1.96 \mathrm{TeV}$, for three different jet algorithms, the anti- $k_{T}, k_{T}$ and the Cambridge/Aachen jet algorithm. The two NLO results refer to different values of the dipole phase space cutoff $\alpha_{\max }$. The scale choice is $\mu_{R}=\mu_{F}=m_{t}$.

$\Lambda_{5}^{M S}=226 \mathrm{MeV}$. In the renormalization of the strong coupling constant, the top-quark loop in the gluon self-energy is subtracted at zero momentum. In this scheme the running of $\alpha_{s}$ is generated solely by the contributions of the light-quark and gluon loops. By default, we set the renormalization and factorization scales, $\mu_{R}$ and $\mu_{F}$, to the common value $\mu=m_{t}$. For inclusive cuts, where the contribution from the double resonance Feynman diagrams dominates, the top mass is a valid scale.

\subsection{Results for the TeVatron run II}

We begin our presentation of the final results of our analysis with a discussion of the total cross section at the central value of the scale, $\mu_{R}=\mu_{F}=m_{t}$ at the TeVatron run II. The respective numbers are presented in table 1 for the two choices of the dipole phase space cutoff parameter $\alpha_{\max }$ (see e.g. [42] for more details) and for three different jet algorithms. At the central scale value, the full cross section receives small NLO correction of the order of $2.3 \%$.

Subsequently, we turn our attention to the scale dependence for the total cross section at LO and NLO. The left panel of figure 3 shows the dependence of the integrated LO cross section on the renormalization and factorization scales where $\mu=\mu_{R}=\mu_{F}=\xi m_{t}$. The variation range is taken from $\mu=m_{t} / 8$ to $\mu=8 m_{t}$. The dependence is large, illustrating the well known fact that the LO prediction can only provide a rough estimate. At the TeVatron with our cut selection the $q \bar{q}$ channel dominates the total $p \bar{p}$ cross section by about $95 \%$ followed by the $g g$ channel with about $5 \%$. In the right panel the scale dependence of the NLO cross section is shown together with the LO one. As expected, we observe a reduction of the scale uncertainty while going from LO to NLO. Varying the scale down and up by a factor 2 changes the cross section by $+40 \%$ and $-26 \%$ in the LO case, while in the NLO case we have obtained a variation of the order $-8 \%$ and $-4 \%$. Let us mention here that while calculating the scale dependence for the NLO cross section we kept $\Gamma_{t}^{N L O}$ fixed independently of the scale choice. The error introduced by this treatment is however of higher order, and particularly for two scales $\mu=m_{t} / 2$ and $\mu=2 m_{t}$ amounts to $\pm 1.5 \%$ respectively.

In the following we would like to estimate the size of the non-factorizable corrections for our inclusive setup. To achieve this the full result has been compared with the result 

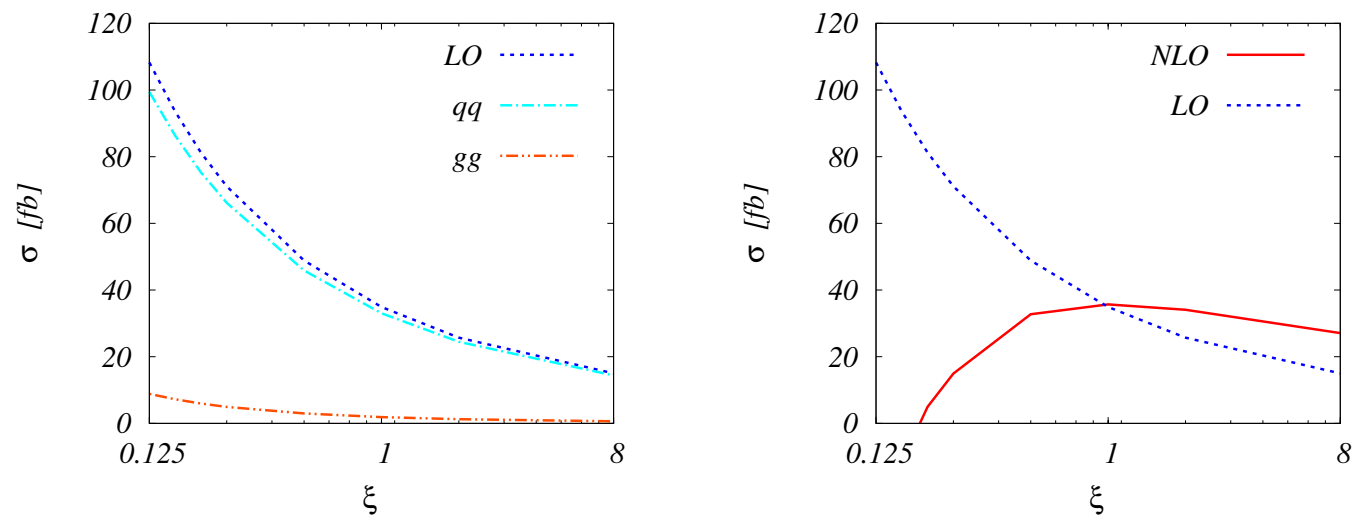

Figure 3. Scale dependence of the LO cross section with the individual contributions of the partonic channels (left panel) and scale dependence of the LO and NLO cross sections (right panel) for the $p \bar{p} \rightarrow e^{+} \nu_{e} \mu^{-} \bar{\nu}_{\mu} b \bar{b}+X$ process at the TeVatron run II with $\sqrt{s}=1.96 \mathrm{TeV}$, where renormalization and factorization scales are set to the common value $\mu=\mu_{R}=\mu_{F}=\xi m_{t}$.

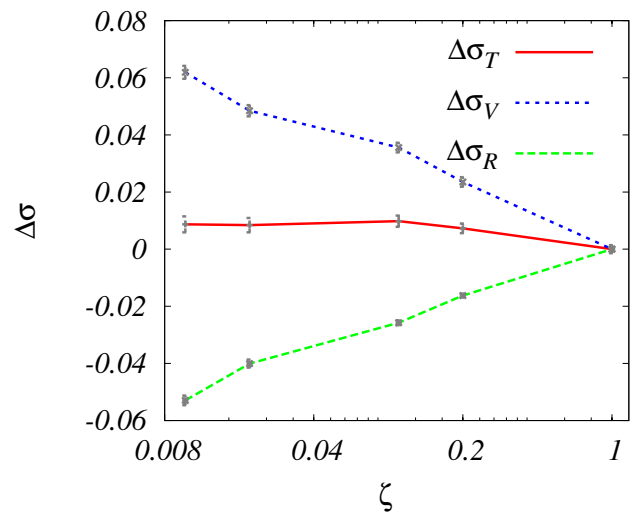

Figure 4. Dependence of the NLO cross section, $\sigma_{\mathrm{T}}$, (red solid line) and the individual contributions, the real emission part, $\sigma_{\mathrm{R}}$, (green dashed line) and the LO plus virtual part, $\sigma_{\mathrm{V}}$, (blue dotted line), on the rescaling parameter $\zeta$ defined as $\Gamma_{\text {rescaled }}=\zeta \Gamma_{t}$ for the $p \bar{p} \rightarrow e^{+} \nu_{e} \mu^{-} \bar{\nu}_{\mu} b \bar{b}+X$ process at the TeVatron run II with $\sqrt{s}=1.96 \mathrm{TeV} . \Delta \sigma$ is defined as follows: $\Delta \sigma_{i}(\zeta)=\left(\sigma_{i}(\zeta)-\sigma_{i}(\zeta=\right.$ $1)) / \sigma_{\mathrm{T}}(\zeta=1)$ with $i=V, R, T$.

in the NWA. The latter has been obtained by rescaling the coupling of the top quark to the $W$ boson and the $\mathrm{b}$ quark by several large factors to mimic the limit $\Gamma_{t} \rightarrow 0$ when the scattering cross section factorizes into on-shell production and decay. Our findings are depicted in figure 4 where the dependence of the total NLO cross section together with its individual contributions, real emission part and LO plus virtual corrections, are shown. The behavior is compatible with a logarithmic dependence on $\Gamma_{t}$, which cancels between real and virtual corrections. For inclusive production, advancing from NWA to the full result changes the cross section no more than $+1 \%$ which is consistent with the uncertainty of the NWA i.e. of order $\mathcal{O}\left(\Gamma_{t} / m_{t}\right)$.

Comparing our NLO integrated cross section with the value $\sigma_{\mathrm{NLO}}=36.47 \mathrm{fb}$ presented in ref. [8], we observe a $2 \%$ discrepancy, which can easily be explained by two effects. First of 

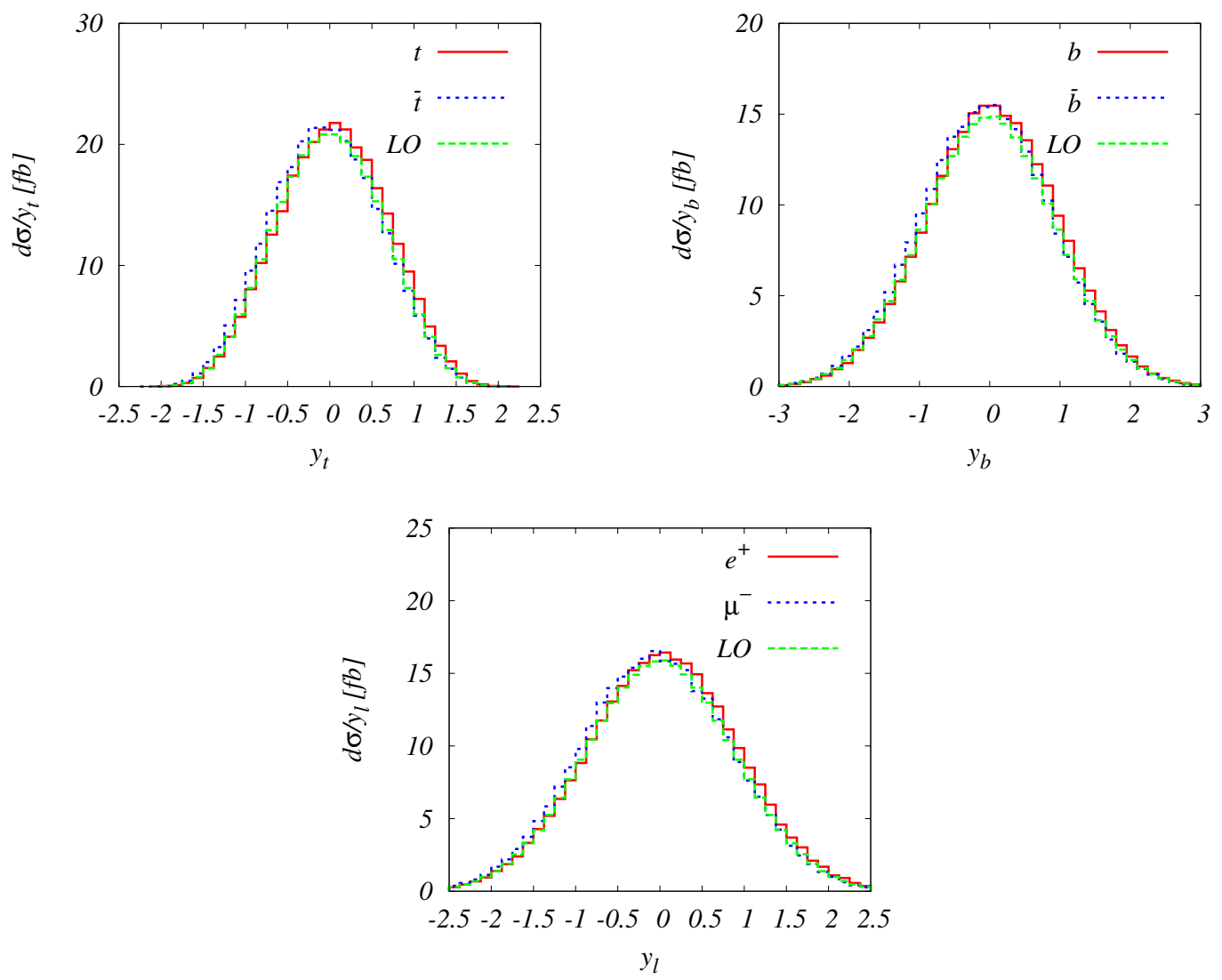

Figure 5. Differential cross section distributions as a function of rapidity $y_{t}$ of the top (red solid curve) and anti-top quarks (blue dotted curve), rapidity $y_{b}$ of the b-jet (red solid curve) and antib-jet (blue dotted curve) and rapidity $y_{l}$ of the positron (red solid curve) and muon (blue dotted curve) at next-to-leading order for the $p p \rightarrow e^{+} \nu_{e} \mu^{-} \bar{\nu}_{\mu} b \bar{b}+X$ process at the TeVatron run II. The green dashed curves correspond to the leading order results.

all, in [8] NLO QCD corrections have been calculated employing an on-shell approximation for the top quarks and the $W$ bosons. The former approximation can introduce a difference of the order of $\mathcal{O}\left(\Gamma_{t} / m_{t}\right) \sim 1 \%$ while the latter of the order of $\mathcal{O}\left(\Gamma_{W} / m_{W}\right) \sim 3 \%$. As a second effect, there are small differences between individual setups, in e.g. the value of $\Gamma_{t}$, $m_{t}, p_{T_{m i s s}}$ and $\Delta R_{j \ell}$.

We have also compared our results with those generated with MCFM. We have been able to use the same cuts and input parameters, but there is an essential difference as far as the construction of the cross section is concerned. Indeed, MCFM includes corrections to the production of on-shell top quarks only, whereas decays are included at leading order. Moreover, $W$ bosons are also treated in the narrow width approximation. In the end, MCFM gives the following results $\sigma_{\mathrm{LO}}=(36.494 \pm 0.050) \mathrm{fb}$ and $\sigma_{\mathrm{NLO}}=(39.622 \pm 0.065) \mathrm{fb}$, which are different from ours by $4.5 \%$ at LO and by $11 \%$ at NLO. Although we have not quantified the impact of different approaches used, related to the top quark and the W-boson finite width, as well as the NLO corrections to the decay of the top quarks, the overall comparison 

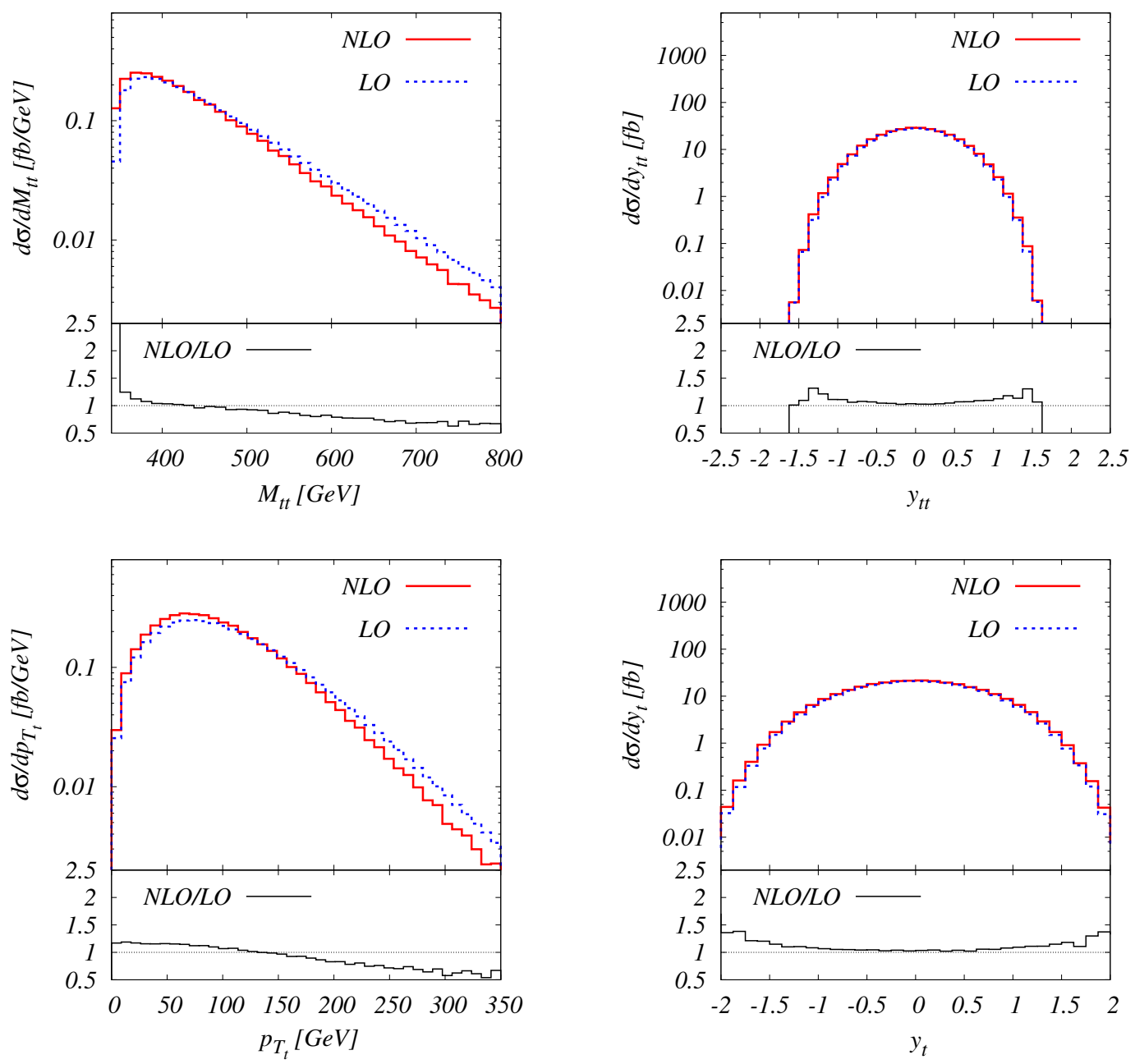

Figure 6. Differential cross section distributions as a function of the invariant mass $m_{t \bar{t}}$ of the top-anti-top pair, rapidity $y_{t \bar{t}}$ of the top-anti-top pair, averaged transverse momentum $p_{T_{t}}$ of the top and anti-top and averaged rapidity $y_{t}$ of the top and anti-top for the $p \bar{p} \rightarrow e^{+} \nu_{e} \mu^{-} \bar{\nu}_{\mu} b \bar{b}+X$ process at the TeVatron run II. The blue dashed curve corresponds to the leading order, whereas the red solid one to the next-to-leading order result. The lower panels display the differential $\mathrm{K}$ factor.

seems reasonable and compatible with estimates based on the order of magnitude for these effects, $\mathcal{O}\left(\Gamma_{t} / m_{t}\right), \mathcal{O}\left(\Gamma_{W} / m_{W}\right)$ and $\mathcal{O}\left(\alpha_{s}\right)$. A more detailed study would be necessary in order to establish the relevance of these differences for the experimental analysis, which goes beyond the purpose of the present publication.

In a next step we recalculate the top quark forward-backward asymmetry for the TeVatron from the top rapidity distribution. We show our results for the LO and NLO inclusive calculations. At LO, tz production is totally charge-conjugation symmetric for both production mechanisms (quark and gluon fusion). As a consequence, the angular distributions of the $t$ and $\bar{t}$ are symmetric with respect to the beam axis for $p \bar{p}$ collisions. However, at higher orders in $\alpha_{s}$, this is not longer true. Not all processes involving additional partons are symmetric under charge conjugation with respect to the incoming parton and 

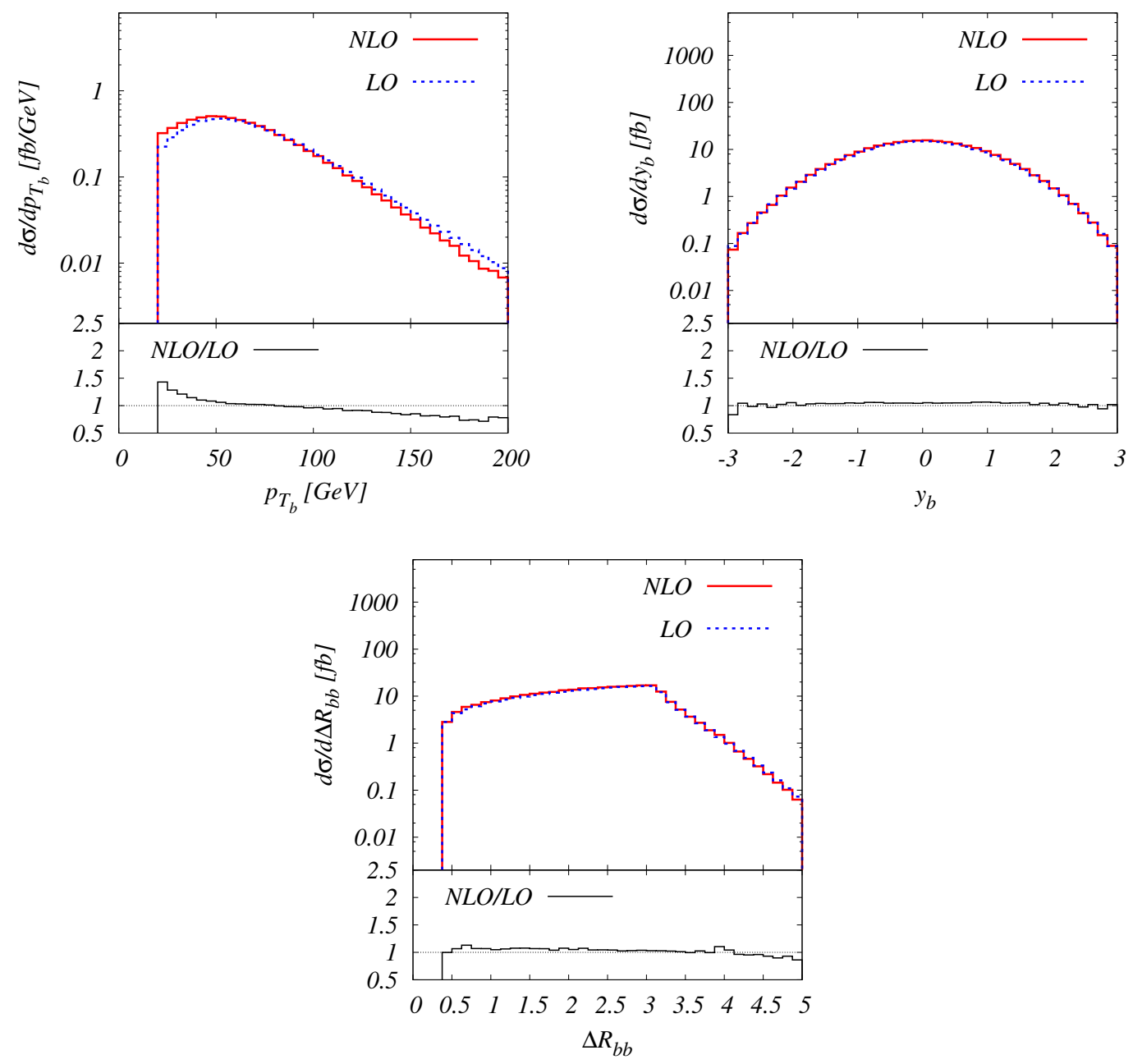

Figure 7. Differential cross section distributions as a function of the averaged transverse momentum $p_{T_{b}}$ of the b-jet and anti-b-jet, averaged rapidity $y_{b}$ of the b-jet and anti-b-jet and $\Delta R_{b \bar{b}}$ separation for the $p \bar{p} \rightarrow e^{+} \nu_{e} \mu^{-} \bar{\nu}_{\mu} b \bar{b}+X$ process at the TeVatron run II. The blue dashed curve corresponds to the leading order, whereas the red solid one to the next-to-leading order result. The lower panels display the differential $\mathrm{K}$ factor.

anti-parton beams. As was pointed out in ref. [77-79] the process $g g \rightarrow t \bar{t} g$ is, but the processes $q \bar{q} \rightarrow t \bar{t} g$ and $q g \rightarrow t \bar{t} q$ are not. Processes involving initial state valence quarks will therefore exhibit a charge asymmetry. This is caused by interference between initial and final state gluon emission on the one side and by interference between color singlet 4 -point virtual corrections and the Born term for the $q \bar{q}$ process $[1,3]$ on the other. Because $t \bar{t}$ production at the TeVatron is dominated at the $95 \%$ level by $q \bar{q}$ annihilation, as was mentioned earlier in the paper, we can expect the $q \bar{q}$ subprocess asymmetry to be visible in the total sample. The integrated charge asymmetry is defined through

$$
A=\frac{\int_{y_{t}>0} N_{t}(y)-\int_{y_{\bar{t}}>0} N_{\bar{t}}(y)}{\int_{y_{t}>0} N_{t}(y)+\int_{y_{\bar{t}}>0} N_{\bar{t}}(y)},
$$



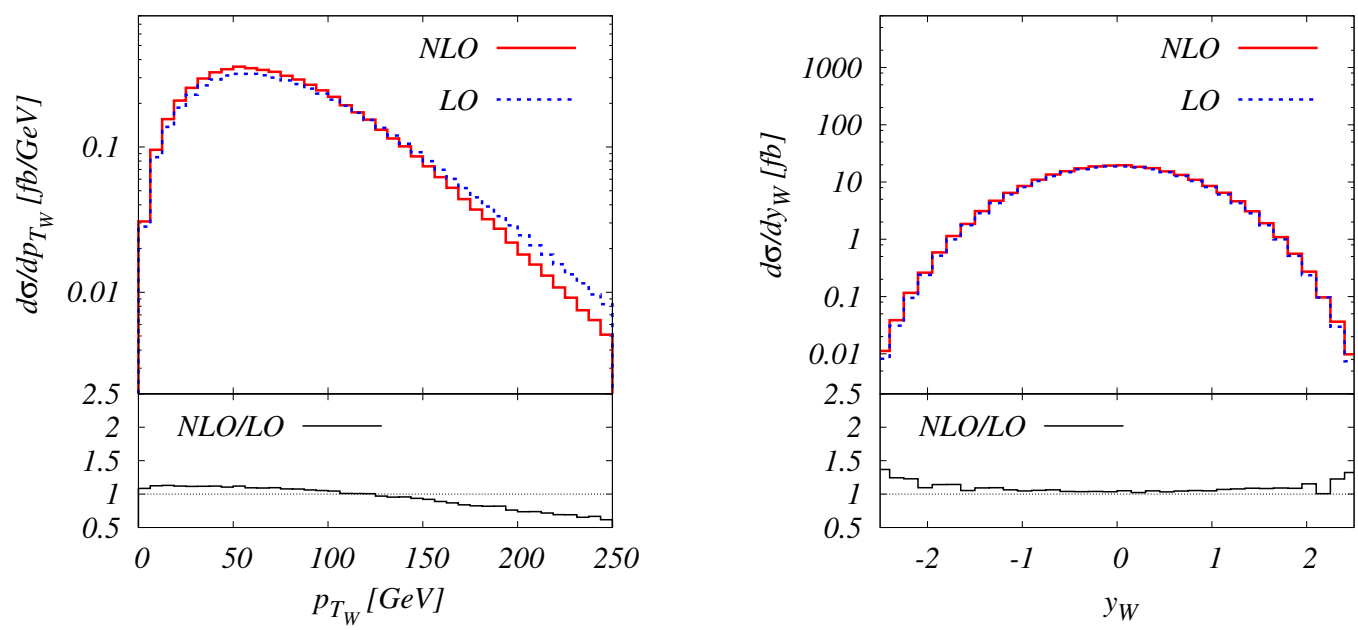

Figure 8. Differential cross section distributions as a function of the averaged transverse momentum $p_{T_{W}}$ of the $W^{ \pm}$bosons and averaged rapidity $y_{W}$ of the $W^{ \pm}$bosons for the $p \bar{p} \rightarrow$ $e^{+} \nu_{e} \mu^{-} \bar{\nu}_{\mu} b \bar{b}+X$ process at the TeVatron run II. The blue dashed curve corresponds to the leading order, whereas the red solid one to the next-to-leading order result. The lower panels display the differential $\mathrm{K}$ factor.

where $y_{t}\left(y_{\bar{t}}\right)$ is the rapidity of the top (anti-top) quark in the laboratory frame and $N_{t}(y)=$ $d \sigma_{t \bar{t}} / d y_{t}, N_{\bar{t}}(y)=d \sigma_{t \bar{t}} / d y_{\bar{t}}$. Due to the $\mathcal{C} \mathcal{P}$ invariance of QCD the rapidity distributions of top and anti-top are mirror images of each other, i.e. $N_{\bar{t}}(y)=N_{t}(-y)$, and integrated charge asymmetry is equal to the integrated forward-backward asymmetry of the top quark defined as

$$
A_{F B}^{t}=\frac{\int_{y>0} N_{t}(y)-\int_{y<0} N_{t}(y)}{\int_{y>0} N_{t}(y)+\int_{y<0} N_{t}(y)} .
$$

Moreover, $A_{F B}^{\bar{t}}=-A_{F B}^{t}$.

As can be seen in the upper-left part of the figure 5 the LO $t \bar{t}$ inclusive cross section is symmetric around $y_{t}=0$ (green dashed curve). The NLO inclusive result for the top/antitop quark is, on the other hand, shifted to larger $y_{t}$ for the top quark (solid red curve) and smaller $y_{t}$ for the anti-top quark (dotted blue curve). This corresponds to a positive integrated forward-backward asymmetry of the order of

$$
A_{F B}^{t}=0.051 \pm 0.0013
$$

which tells us that top quarks are preferentially emitted in the direction of the incoming protons.

Next-to-leading order contributions to the forward-backward asymmetry have already been calculated in the on-shell $t \bar{t}$ production [80] and amount to $A_{F B}^{t}=0.051 \pm 0.006$. The $\mathrm{CDF}$ measurement based on $5.3 \mathrm{fb}^{-1}$ integrated luminosity in the semi-leptonic channel yields $A_{F B}^{t}=0.150 \pm 0.050^{\text {stat. }} \pm 0.024^{\text {syst. }}$ [81], while the $\mathrm{D} \varnothing$ measurement of this asymmetry yields $A_{F B}^{t}=0.08 \pm 0.04^{\text {stat. }} \pm 0.01^{\text {syst. }}$ based on $4.3 \mathrm{fb}^{-1}$ integrated luminosity [82]. The uncertainties of these results are still very large and statistically dominated. 

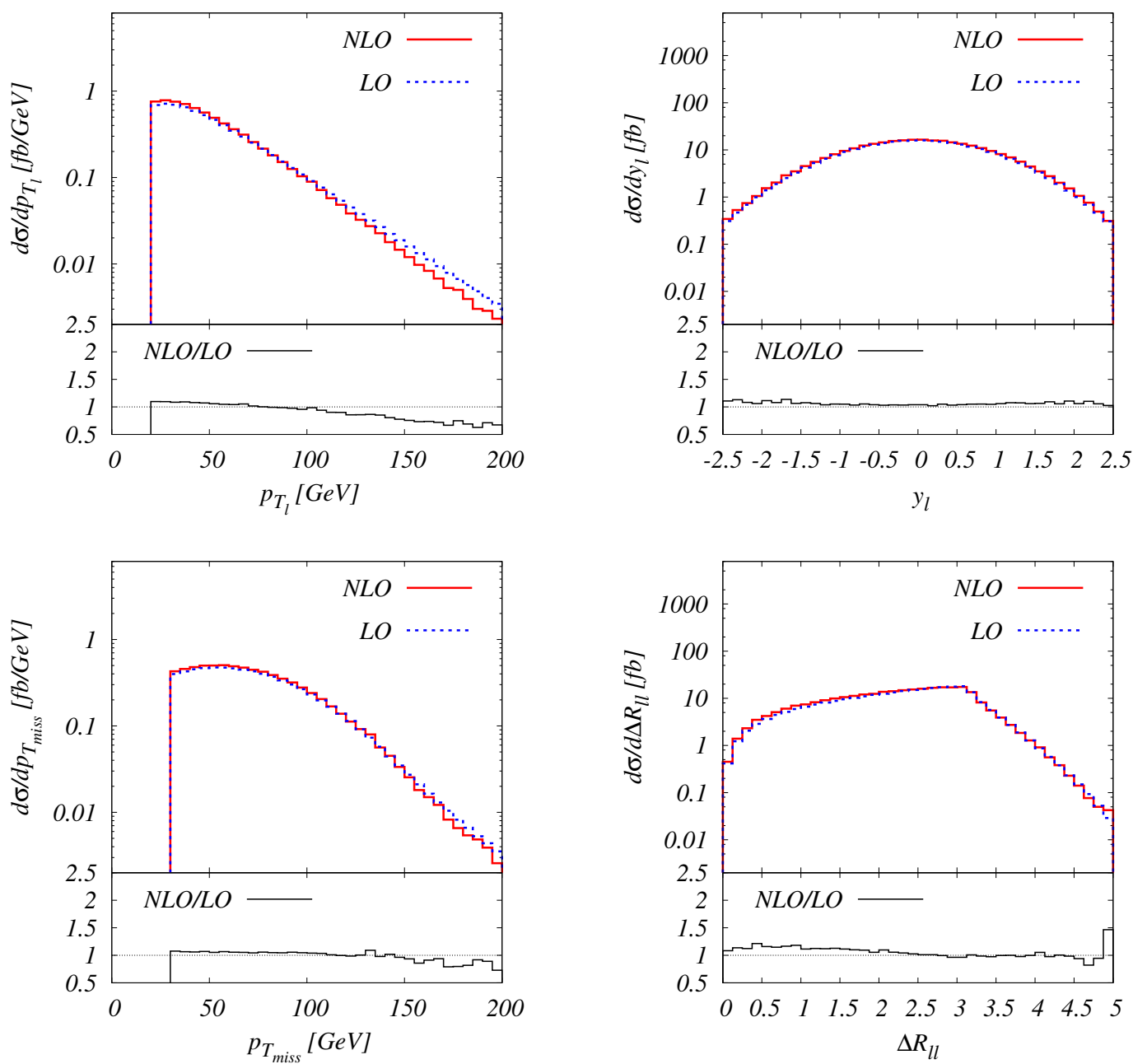

Figure 9. Differential cross section distributions as a function of the averaged transverse momentum $p_{T_{\ell}}$ of the charged leptons, averaged rapidity $y_{\ell}$ of the charged leptons, $p_{T_{m i s s}}$ and $\Delta R_{\ell \ell}$ for the $p \bar{p} \rightarrow e^{+} \nu_{e} \mu^{-} \bar{\nu}_{\mu} b \bar{b}+X$ process at the TeVatron run II. The blue dashed curve corresponds to the leading order, whereas the red solid one to the next-to-leading order result. The lower panels display the differential $\mathrm{K}$ factor.

In the same manner we can calculate the integrated forward-backward asymmetry for the top decay products, namely the $b$-jet and the positively charged lepton. Our results can be summarized as follows:

$$
A_{F B}^{b}=0.033 \pm 0.0013, \quad A_{F B}^{\ell^{+}}=0.034 \pm 0.0013,
$$

where

$$
A_{F B}^{b}=\frac{\int_{y_{b}>0} N_{b}(y)-\int_{y_{b}<0} N_{b}(y)}{\int_{y_{b}>0} N_{b}(y)+\int_{y_{b}<0} N_{b}(y)}, \quad A_{F B}^{\ell^{+}}=\frac{\int_{y_{\ell^{+}}>0} N_{\ell^{+}}(y)-\int_{y_{\ell^{+}}<0} N_{\ell^{+}}(y)}{\int_{y_{\ell^{+}}>0} N_{\ell^{+}}(y)+\int_{y_{\ell^{+}}<0} N_{\ell^{+}}(y)}
$$

and $y_{\ell}$ and $y_{b}$ are the rapidity of the charged lepton and the $b$-jet respectively and $N_{\ell^{+}}(y)=$ $d \sigma_{t \bar{t}} / d y_{\ell^{+}}, N_{b}(y)=d \sigma_{t \bar{t}} / d y_{b}$. In case of $A_{F B}^{\ell^{+}}$we agree with ref. [7] where $A_{F B}^{\ell^{+}}=0.033$ has 


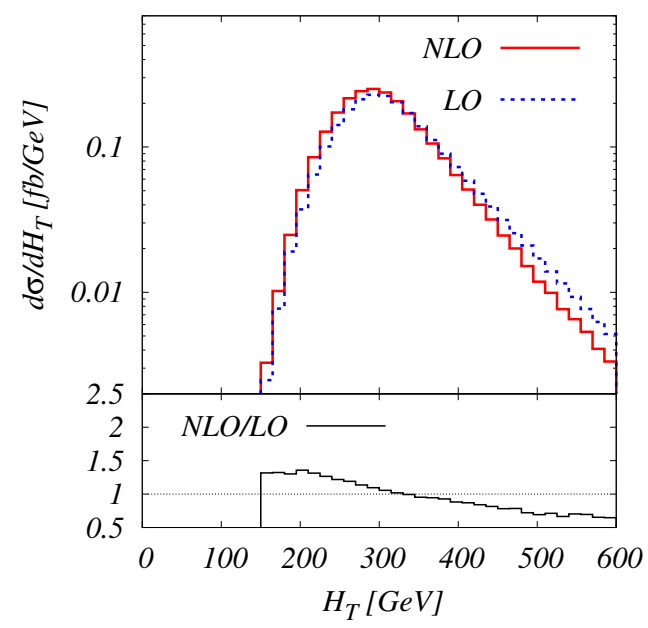

Figure 10. Differential cross section distribution as a function of the total transverse energy, $H_{T}$, for the $p \bar{p} \rightarrow e^{+} \nu_{e} \mu^{-} \bar{\nu}_{\mu} b \bar{b}+X$ process at the TeVatron run II. The blue dashed curve corresponds to the leading order, whereas the red solid one to the next-to-leading order result. The lower panels display the differential $\mathrm{K}$ factor.

been quoted. The integrated forward-backward asymmetries of the charged lepton and the $b$-jet have the same sign as $A_{F B}^{t}$ but are smaller in magnitude. Let us stress at this point, that the $b$-jet integrated forward-backward asymmetry is a rather theoretical observable even though it can in principle be measured once the $b$-jet is distinguished experimentally from the anti-b-jet through e.g. the charge of the associated lepton flying in the same direction. However, it is extremely difficult to determine the charge of the $b$-jet and this measurement will heavily depend on the $b$-jet tagging efficiency. The $b$-jet and charged lepton differential distributions in rapidity are also presented in figure 5.

While the size of the corrections to the total cross section is certainly interesting, it is crucial to study the corrections to distributions. In the following, the NLO QCD corrections to the differential distributions for the dileptonic channel with full off-shell effects are presented.

In figure 6 we start with the most important observable, namely, the differential distribution of the $t \bar{t}$ invariant mass, $m_{t \bar{t}}$. Figure 6 depicts also the rapidity, $y_{t \bar{t}}$, of the top-anti-top system as well as the averaged transverse momentum, $p_{T_{t}}$, and the averaged rapidity $y_{t}$ of the top and anti-top. The blue dashed curve corresponds to the leading order, whereas the red solid one to the next-to-leading order result. The histograms can also be turned into dynamical K-factors, which we display in the lower panels. The small size of the corrections to the total cross section is reflected only in the angular distributions, where we can see positive corrections of the order of $5 \%-10 \%$. Both distributions of $m_{t \bar{t}}$ and $p_{T_{t}}$ get sizeable negative corrections for large values of these observables. For the $m_{t \bar{t}}$ distribution, corrections reach $-30 \%$ which has to be compared with positive $+25 \%$ corrections close to the $t \bar{t}$ threshold. The $p_{T_{t}}$ distribution is corrected down to $-40 \%$ at the tails and $+20 \%$ for small values of $p_{T_{t}}$. Overall, this leads to a distortion of the differential distributions up to $55 \%-60 \%$. Given that top-quark pair production at high scale is an ideal tool to search 
for various models of physics beyond the Standard Model with new gauge bosons like e.g. $Z^{\prime}$, it is clear that a precise knowledge of the higher order corrections in this region is of significant importance.

In figure 7 , the $b$-jet kinematics is presented, where differential cross section distributions as a function of the averaged transverse momentum, $p_{T_{b}}$, and averaged rapidity, $y_{b}$, of the $b$ - and anti-b-jet are presented together with the $\Delta R_{b \bar{b}}$ separation. Both angular distributions, $y_{b}$ and $\Delta R_{b \bar{b}}$, exhibit small positive corrections $5 \%-10 \%$, however, for the $p_{T_{b}}$ distribution we observe large and positive corrections of the order of $+30 \%$ at the begin of the spectrum and negative of the order of $-20 \%$ around $200 \mathrm{GeV}$.

A similar situation is observed for the $W^{ \pm}$boson kinematics which is shown in figure 8, where the differential cross section distributions as function of the averaged transverse momentum $p_{T_{W}}$ of the $W^{ \pm}$bosons together with an averaged rapidity $y_{W}$ of the $W^{ \pm}$ bosons are depicted. Yet again, small positive corrections of $5 \%-10 \%$ are acquired for angular distributions as well as for low values of $p_{T_{W}}$, while the tail of the $p_{T_{W}}$ differential distribution exhibits negative corrections down to $-30 \%$.

Subsequently, in figure 9, differential cross section distributions as function of the averaged transverse momentum $p_{T_{\ell}}$ and averaged rapidity $y_{\ell}$ of the charged leptons together with $p_{T_{m i s s}}$ and the separation $\Delta R_{\ell \ell}$ are shown. Also here, a distortion of the $p_{T_{\ell}}$ differential distribution up to $40 \%$ is reached, while for $p_{T_{m i s}}$ up to $15 \%$. For the angular distributions, moderate corrections up to $+10 \%$ are obtained.

And finally, in figure 10, the differential cross section distribution as function of the total transverse energy defined as

$$
H_{T}=p_{T_{b}}+p_{T_{\bar{b}}}+p_{T_{e^{+}}}+p_{T_{\mu^{-}}}+p_{T_{m i s s}}
$$

is presented. In this case we observe a distortion of the differential distribution up to $70 \%-80 \%$.

Overall, we can say that at the TeVatron, employing a fixed scale $\mu=m_{t}$, the NLO corrections to transverse momentum distributions are moderate. However, they do not simply rescale the LO shapes, but induce distortions at the level of $15 \%-80 \%$, which redistribute events from larger to smaller transverse momenta. The same applies to the invariant mass distribution of the $t \bar{t}$ pair. As for angular distributions we observe positive and rather modest corrections of the order of $5 \%-10 \%$.

\subsection{Results for the $\mathrm{LHC}$}

Table 2 shows the integrated cross sections at the LHC with $\sqrt{s}=7 \mathrm{TeV}$, for two choices of the $\alpha_{\max }$ parameter and for three different jet algorithms. At the central scale value, the full cross section receives NLO QCD corrections of the order of 47\%. Figure 11 presents the dependence of the integrated LO cross section on the renormalization and factorization scales where $\mu=\mu_{R}=\mu_{F}=\xi m_{t}$. The variation range from $\mu=m_{t} / 8$ to $\mu=8 m_{t}$. In contrast to the TeVatron, the gg channel comprises about $76 \%$ of the LO $p p$ cross section, followed by the $q \bar{q}$ channel with about $24 \%$. In the right panel of figure 11, the scale dependence of the NLO cross section is shown together with the LO one. Comparing the 


\begin{tabular}{|c|c|c|c|}
\hline \multirow{2}{*}{ Algorithm } & $\sigma_{\mathrm{LO}}[\mathrm{fb}]$ & $\sigma_{\mathrm{NLO}}^{\alpha_{\max }=1}[\mathrm{fb}]$ & $\sigma_{\mathrm{NLO}}^{\alpha_{\max }=0.01}[\mathrm{fb}]$ \\
\hline${\text { anti- } k_{T}}$ & $550.54 \pm 0.18$ & $808.46 \pm 0.98$ & $808.29 \pm 1.04$ \\
\hline$k_{T}$ & $550.54 \pm 0.18$ & $808.67 \pm 0.97$ & $808.86 \pm 1.03$ \\
\hline $\mathrm{C} / \mathrm{A}$ & $550.54 \pm 0.18$ & $808.74 \pm 0.97$ & $808.28 \pm 1.03$ \\
\hline
\end{tabular}

Table 2. Integrated cross section at LO and NLO for $p p \rightarrow e^{+} \nu_{e} \mu^{-} \bar{\nu}_{\mu} b \bar{b}+X$ production at the LHC with $\sqrt{s}=7 \mathrm{TeV}$, for three different jet algorithms, the anti- $k_{T}, k_{T}$ and for the Cambridge/Aachen jet algorithm. The two NLO results refer to different values of the dipole phase space cutoff $\alpha_{\max }$. The scale choice is $\mu_{R}=\mu_{f}=m_{t}$.
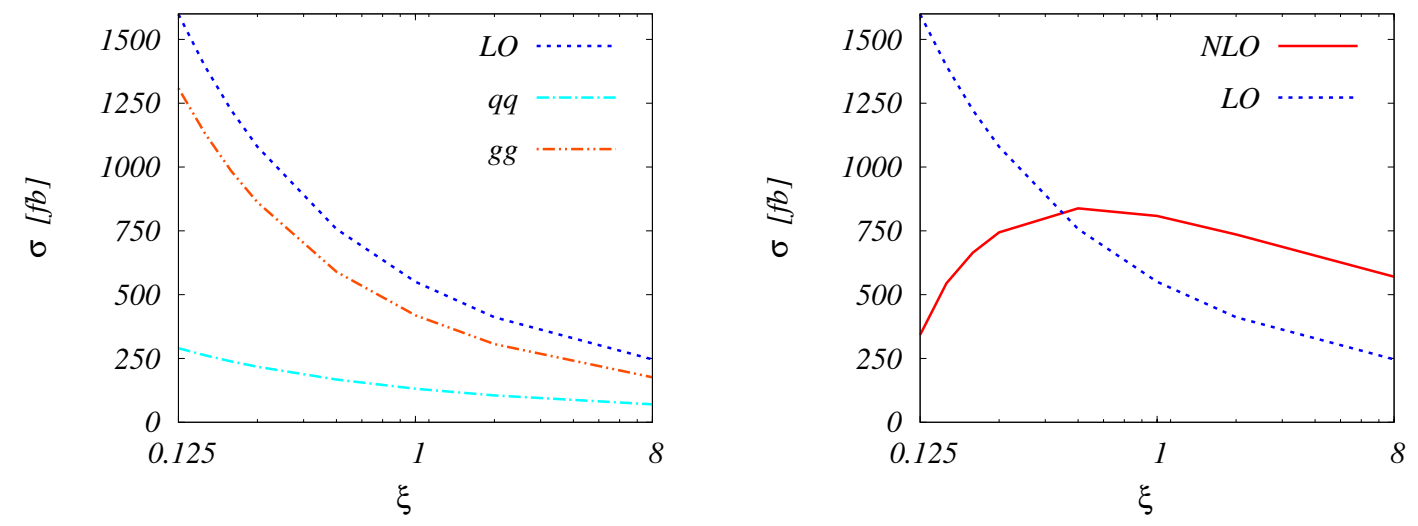

Figure 11. Scale dependence of the LO cross section with the individual contributions of the partonic channels (left panel) and scale dependence of the LO and NLO cross sections (right panel) for the $p p \rightarrow e^{+} \nu_{e} \mu^{-} \bar{\nu}_{\mu} b \bar{b}+X$ process at the LHC with $\sqrt{s}=7 \mathrm{TeV}$, where renormalization and factorization scales are set to the common value $\mu=\mu_{R}=\mu_{F}=\xi m_{t}$.

\begin{tabular}{|c|c|c|c|}
\hline \multirow{2}{*}{ Algorithm } & $\sigma_{\mathrm{LO}}[\mathrm{fb}]$ & $\sigma_{\mathrm{NLO}}^{\alpha_{\max }=1}[\mathrm{fb}]$ & $\sigma_{\mathrm{NLO}}^{\alpha_{\max }=0.01}[\mathrm{fb}]$ \\
\hline${\text { anti- } k_{T}}$ & $1394.72 \pm 0.75$ & $1993.3 \pm 2.5$ & $1993.9 \pm 2.7$ \\
\hline$k_{T}$ & $1394.72 \pm 0.75$ & $1995.2 \pm 2.5$ & $1994.3 \pm 2.7$ \\
\hline $\mathrm{C} / \mathrm{A}$ & $1394.72 \pm 0.75$ & $1995.0 \pm 2.5$ & $1994.3 \pm 2.7$ \\
\hline
\end{tabular}

Table 3. Integrated cross section at LO and NLO for $p p \rightarrow e^{+} \nu_{e} \mu^{-} \bar{\nu}_{\mu} b \bar{b}+X$ production at the LHC with $\sqrt{s}=10 \mathrm{TeV}$, for three different jet algorithms, the anti- $k_{T}, k_{T}$ and for the Cambridge/Aachen jet algorithm. The two NLO results refer to different values of the dipole phase space cutoff $\alpha_{\max }$. The scale choice is $\mu_{R}=\mu_{f}=m_{t}$.

LO and NLO predictions, we find again that the large scale dependence of about $+37 \%$ and $-25 \%$ in the LO cross section is considerably reduced, down to $+4 \%$ and $-9 \%$ when varying the scale down and up by a factor 2, after including the NLO corrections.

In order to quantify the size of the non-factorizable corrections for the LHC, we analyze once more the narrow-width limit of our calculation following the procedure described in 


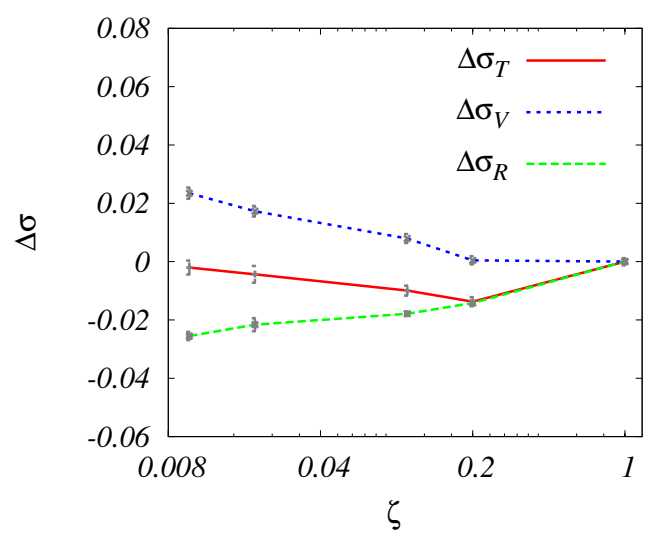

Figure 12. Dependence of the NLO cross section, $\sigma_{\mathrm{T}}$, (red solid line) and the individual contributions, the real emission part, $\sigma_{\mathrm{R}}$, (green dashed line) and the LO plus virtual part, $\sigma_{\mathrm{V}}$, (blue dotted line), on the rescaling parameter $\zeta$ defined as $\Gamma_{\text {rescaled }}=\zeta \Gamma_{t}$ for the $p p \rightarrow e^{+} \nu_{e} \mu^{-} \bar{\nu}_{\mu} b \bar{b}+X$ process at the LHC with $\sqrt{s}=7 \mathrm{TeV} . \Delta \sigma$ is defined as follows: $\Delta \sigma_{i}(\zeta)=\left(\sigma_{i}(\zeta)-\sigma_{i}(\zeta=1)\right) / \sigma_{\mathrm{T}}(\zeta=1)$ with $i=V, R, T$.

section 3.2. Our results are presented in figure 12, where the dependence of the total NLO cross section together with its individual contributions, real emission part and the LO plus virtual corrections, are shown. Also in this case, the behavior is compatible with a logarithmic dependence on $\Gamma_{t}$ which cancels between real and virtual corrections. Going from NWA to the full result changes the cross section no more than $-1.2 \%$ for our inclusive setup, which is within the expected uncertainty of $\mathcal{O}\left(\Gamma_{t} / m_{t}\right)$ of the NWA approach.

In table 3 , the integrated cross sections at the LHC with $\sqrt{s}=10 \mathrm{TeV}$ are presented, once more for two choices of the $\alpha_{\max }$ parameter and for the three different jet algorithms. In this case, at the central scale value, the full cross section receives NLO QCD corrections of the order of $43 \%$.

In a next step, we compare our NLO integrated cross section with the value $\sigma_{\mathrm{NLO}}=$ $2097 \mathrm{fb}$ for $\sqrt{s}=10 \mathrm{TeV}$ presented in ref. [8]. We observe a $5 \%$ discrepancy which can perfectly be explained using the same arguments as in the TeVatron case, namely the on-shell top and $W$ boson approximation applied in [8] and small differences between individual setups.

As in the case of TeVatron, we have also made a comparison with McFM. We have obtained $\sigma_{\mathrm{LO}}=(563.01 \pm 0.63) \mathrm{fb}, \sigma_{\mathrm{NLO}}=(838.98 \pm 1.68) \mathrm{fb}$ for $\sqrt{s}=7 \mathrm{TeV}$ and $\sigma_{\mathrm{LO}}=(1421.05 \pm 1.59) \mathrm{fb}, \sigma_{\mathrm{NLO}}=(2046.9 \pm 4.3) \mathrm{fb}$ for $\sqrt{s}=10 \mathrm{TeV}$, which constitutes a difference of $2 \%$ at LO for both cases and a difference of $4 \%$ and $3 \%$ respectively at NLO. Moreover, both NLO results remain within our theoretical uncertainty of $9 \%$, which is due to scale variation.

Top quark production at the LHC is forward-backward symmetric in the laboratory frame as a consequence of the symmetric colliding proton-proton initial state. Therefore, we turn our attention to the size of NLO QCD corrections to the differential distributions at the LHC. 

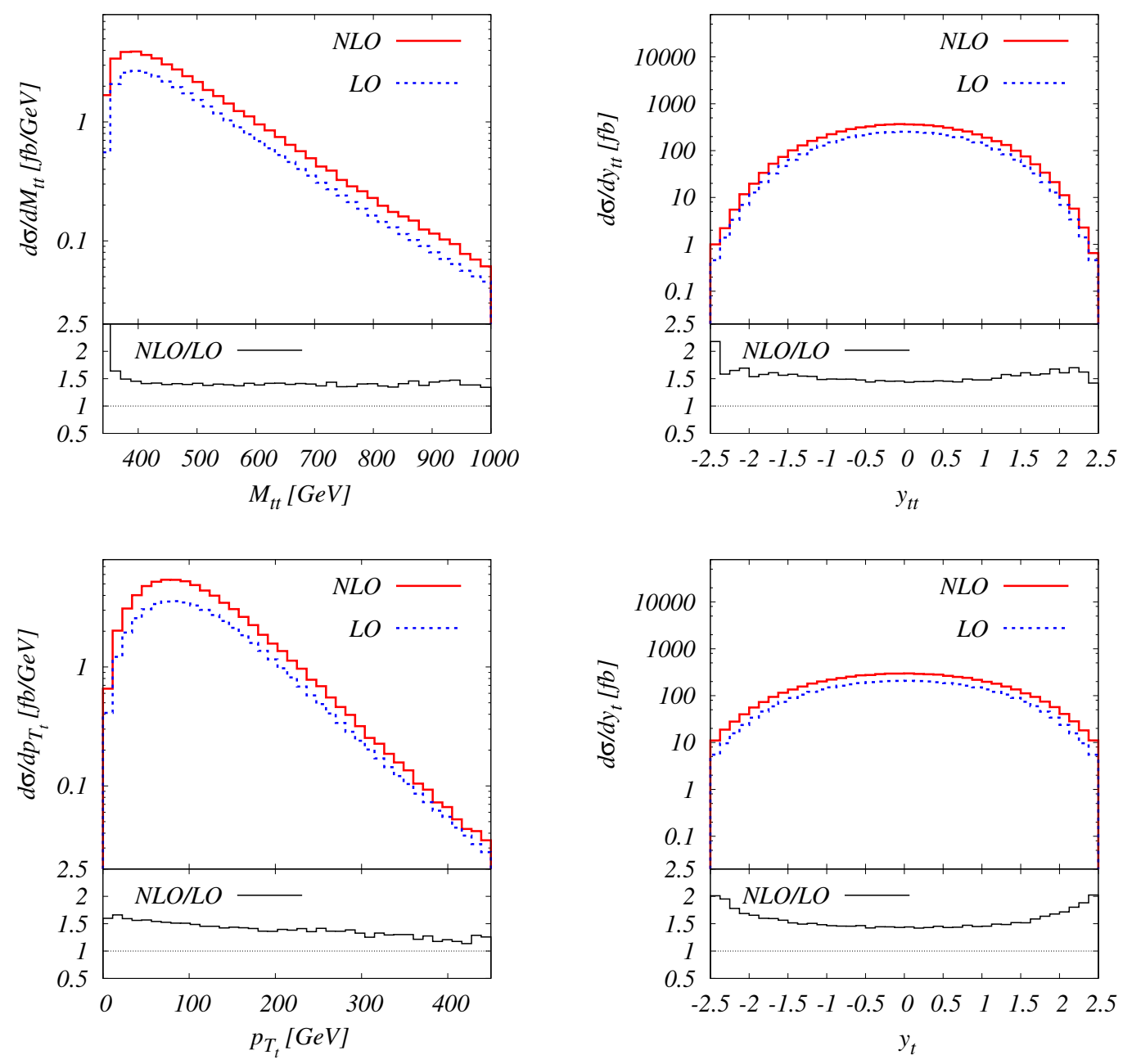

Figure 13. Differential cross section distributions as a function of the invariant mass $m_{t \bar{t}}$ of the top-anti-top pair, rapidity $y_{t \bar{t}}$ of the top-anti-top pair, averaged transverse momentum $p_{T_{t}}$ of the top and anti-top and averaged rapidity $y_{t}$ of the top and anti-top for the $p p \rightarrow e^{+} \nu_{e} \mu^{-} \bar{\nu}_{\mu} b \bar{b}+X$ process at the LHC with $\sqrt{s}=7 \mathrm{TeV}$. The blue dashed curve corresponds to the leading order, whereas the red solid one to the next-to-leading order result. The lower panels display the differential $\mathrm{K}$ factor.

We present the differential distributions only for the $\sqrt{s}=7 \mathrm{TeV}$ case. In figure 13 , differential distributions of the $t \bar{t}$ invariant mass, $m_{t \bar{t}}$, together with the rapidity distribution, $y_{t \bar{t}}$, of the top-anti-top system as well as the averaged transverse momentum, $p_{T_{t}}$, and the averaged rapidity $y_{t}$ of the top and anti-top are depicted. Distributions become harder in $p_{T}$ and in the invariant mass of the $t \bar{t}$ pair, moving from the TeVatron to the LHC case, as expected from the higher scattering energy. Rapidity distributions of the $t \bar{t}$ pair and the $t$ quark, on the other hand, get broadened in this transition. NLO QCD corrections to these differential distributions are always positive and below $50 \%-60 \%$. In case of rapidity distributions this applies for events concentrated within $\left|y_{t \bar{t}}\right|<2$ and $\left|y_{t}\right|<2$ regions.

In figure 14, the $b$-jet kinematics is presented again, but this time in the framework of the LHC. In particular, differential cross section distributions as function of the averaged 

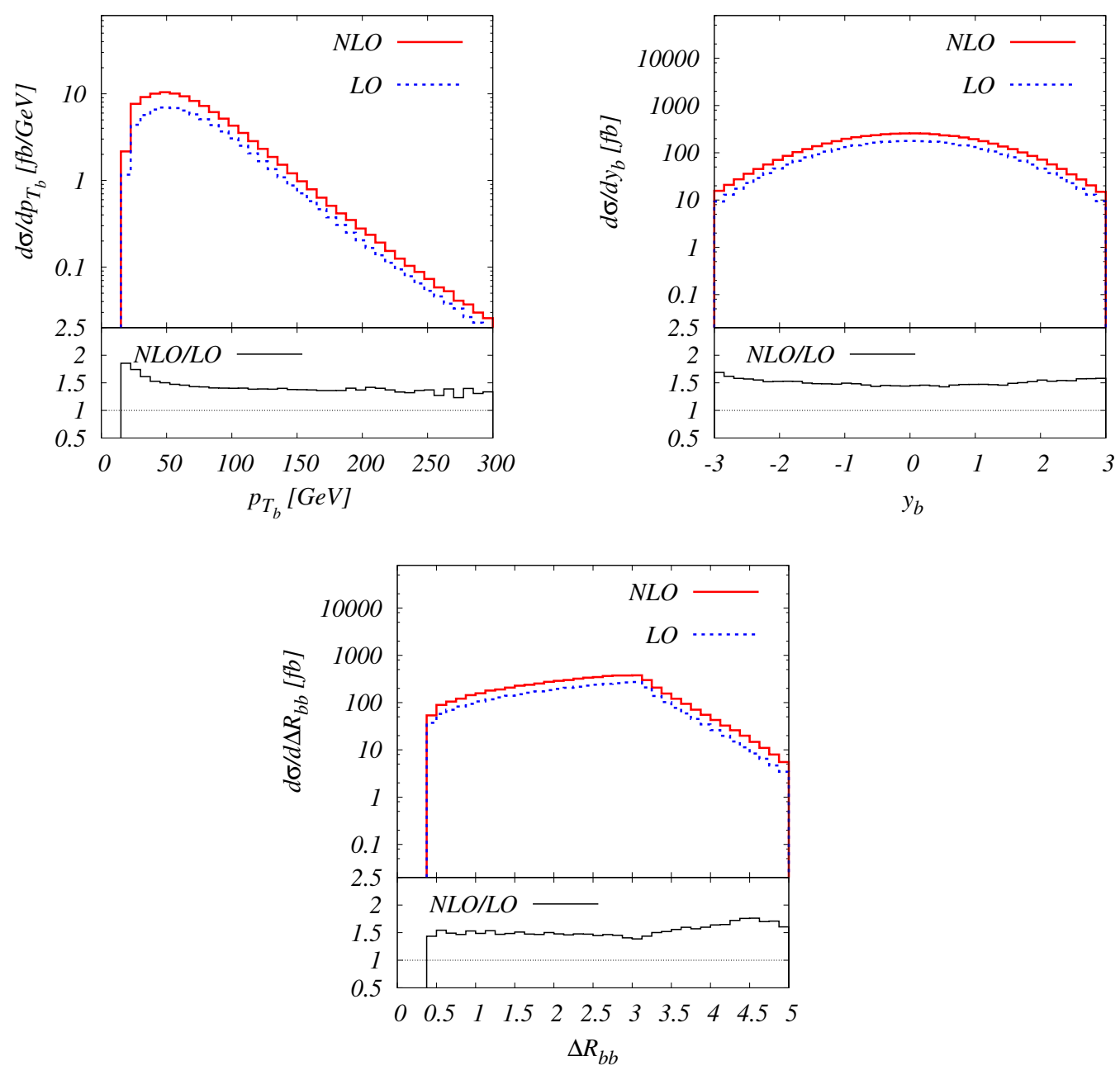

Figure 14. Differential cross section distributions as a function of the averaged transverse momentum $p_{T_{b}}$ of the b-jet, averaged rapidity $y_{b}$ of the b-jet and $\Delta R_{b \bar{b}}$ separation for the $p p \rightarrow e^{+} \nu_{e} \mu^{-} \bar{\nu}_{\mu} b \bar{b}+X$ process at the LHC with $\sqrt{s}=7 \mathrm{TeV}$. The blue dashed curve corresponds to the leading order, whereas the red solid one to the next-to-leading order result. The lower panels display the differential $\mathrm{K}$ factor.

transverse momentum, $p_{T_{b}}$, and averaged rapidity, $y_{b}$, of the $b$ - and anti- $b$-jet are presented together with the $\Delta R_{b \bar{b}}$ separation. Also in this case, the $p_{T_{b}}$ distribution is harder than at the TeVatron and the $y_{b}$ distribution is broader. Clearly, the distributions show the same large and positive corrections, which turn out to be relatively constant. Only in case of $\Delta R_{b \bar{b}}$, corrections lead to a distortion of the differential distributions up to $30 \%$.

The $W^{ \pm}$boson kinematics is shown in figure 15, where the differential cross section distributions as a function of the averaged transverse momentum $p_{T_{W}}$ of the $W^{ \pm}$bosons together with an averaged rapidity $y_{W}$ of the $W^{ \pm}$bosons are depicted. Large positive corrections of $50 \%-60 \%$ are acquired for $p_{T_{W}}$ differential distribution and rapidity distribution with events concentrated within $\left|y_{W^{ \pm}}\right|<2$. The tails of the $y_{W^{ \pm}}$distribution acquire even higher NLO QCD corrections. 

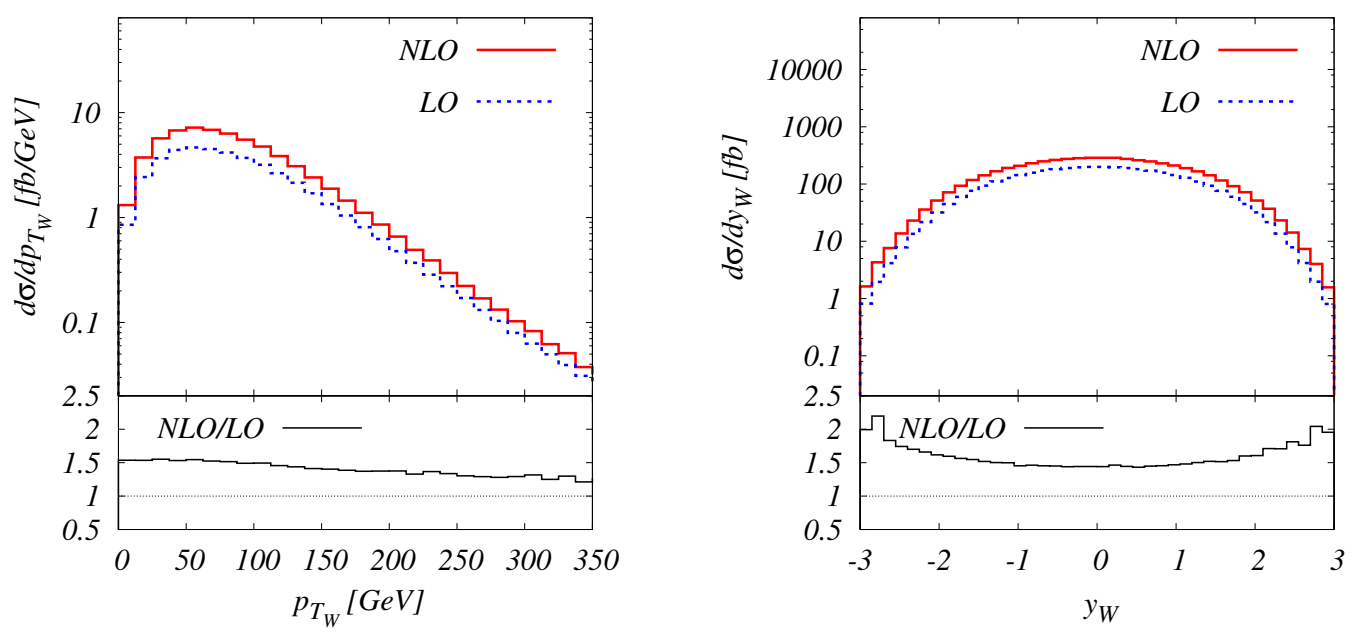

Figure 15. Differential cross section distributions as a function of the averaged transverse momentum $p_{T_{W}}$ of the $W^{ \pm}$bosons and averaged rapidity $y_{W}$ of the $W^{ \pm}$bosons for the $p p \rightarrow$ $e^{+} \nu_{e} \mu^{-} \bar{\nu}_{\mu} b \bar{b}+X$ process at the LHC with $\sqrt{s}=7 \mathrm{TeV}$. The blue dashed curve corresponds to the leading order, whereas the red solid one to the next-to-leading order result. The lower panels display the differential $\mathrm{K}$ factor.

Subsequently, in figure 16, differential cross section distributions as function of the averaged transverse momentum $p_{T_{\ell}}$ and averaged rapidity $y_{\ell}$ of the charged leptons together with $p_{T_{m i s s}}$ and $\Delta R_{\ell \ell}$ separation are shown. A small distortion of the $p_{T_{\ell}}$ differential distribution up to $25 \%$ is reached, while for $p_{T_{m i s s}}$ a distortion up to $70 \%-80 \%$ is visible. For the $y_{\ell}$ distribution, large positive and rather constant corrections up to $50 \%$ are obtained, and for the tails of the $\Delta R_{\ell \ell}$ distribution corrections of $80 \%-90 \%$ are obtained.

Finally, in figure 17 the differential cross section distribution as function of the total transverse energy defined in (3.9) is presented. In this case we observe a distortion of the differential distribution up to $40 \%$.

Generally, we can say that for a fixed scale $\mu=m_{t}$ at LHC, the NLO QCD corrections are always positive and large, at the level of $50 \%-60 \%$. Furthermore, they are relatively constant. Exceptions are the rapidity distributions, which are only constant in the central region, and the $p_{T_{m i s s}}$ and $H_{T}$ distributions, which are distorted up to $40 \%-80 \%$.

\section{Conclusions}

In this paper, we have presented, for the first time, a computation of the NLO QCD corrections to the full decay chain $p p(p \bar{p}) \rightarrow t \bar{t} \rightarrow W^{+} W^{-} b \bar{b} \rightarrow e^{+} \nu_{e} \mu^{-} \bar{\nu}_{\mu} b \bar{b}+X$. All off-shell effects of top quarks and $W$ gauge bosons have been included in a fully differential manner which allows us to compute an arbitrary observable in terms of jets, charged leptons and missing transverse energy within experimentally relevant selection criteria with NLO QCD accuracy. In order to illustrate the capabilities of the program, the total cross section and its scale dependence, as well as several differential distributions at the TeVatron run II and the LHC have been given. Moreover, in case of the TeVatron the forward-backward 

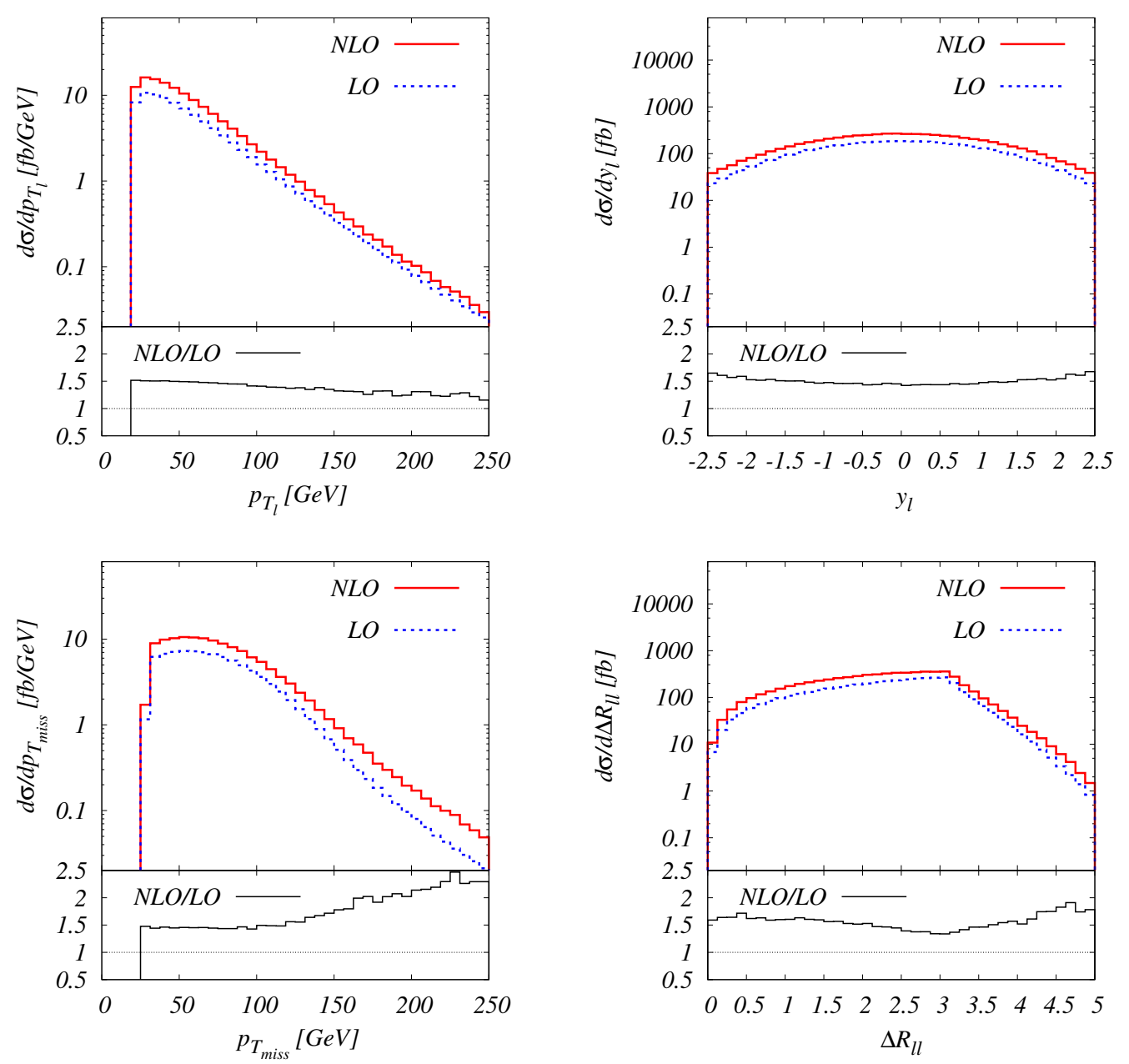

Figure 16. Differential cross section distributions as a function of the averaged transverse momentum $p_{T_{\ell}}$ of the charged leptons, averaged rapidity $y_{\ell}$ of the charged leptons, $p_{T_{m i s s}}$ and $\Delta R_{\ell \ell}$ for the $p p \rightarrow e^{+} \nu_{e} \mu^{-} \bar{\nu}_{\mu} b \bar{b}+X$ process at the LHC with $\sqrt{s}=7 \mathrm{TeV}$. The blue dashed curve corresponds to the leading order, whereas the red solid one to the next-to-leading order result. The lower panels display the differential $\mathrm{K}$ factor.

asymmetry of the top has been recalculated. We have found that with inclusive selection cuts, the forward-backward asymmetry amounts to $A_{F B}^{t}=0.051 \pm 0.0013$. Furthermore, the impact of the NLO QCD corrections on integrated cross sections at the TeVatron is small, of the order $2.3 \%$. At the LHC we have obtained NLO QCD corrections at the level of $47 \%$ and $43 \%$ for $\sqrt{s}=7 \mathrm{TeV}$ and $\sqrt{s}=10 \mathrm{TeV}$ respectively. A study of the scale dependence of our NLO predictions indicates that the residual theoretical uncertainty due to higher order corrections is $8 \%$ for the TeVatron and $9 \%$ for the LHC.

\section{Acknowledgments}

We would like to thank John Campbell and Keith Ellis for their help in using MCFM.

The work was funded in part by the RTN European Programme MRTN-CT-2006035505 HEPTOOLS - Tools and Precision Calculations for Physics Discoveries at Colliders. 


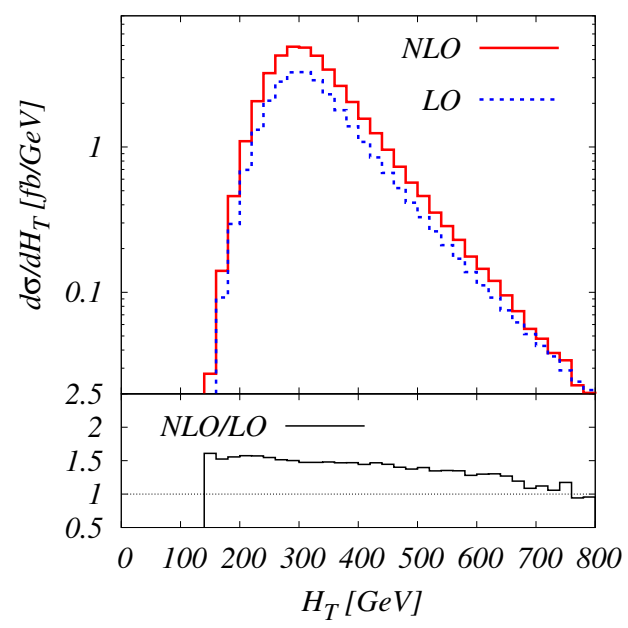

Figure 17. Differential cross section distribution as a function of the total transverse energy, $H_{T}$, for the $p p \rightarrow e^{+} \nu_{e} \mu^{-} \bar{\nu}_{\mu} b \bar{b}+X$ process at the LHC with $\sqrt{s}=7 \mathrm{TeV}$. The blue dashed curve corresponds to the leading order, whereas the red solid one to the next-to-leading order result. The lower panels display the differential $\mathrm{K}$ factor.

M. Czakon was supported by the Heisenberg and by the Gottfried Wilhelm Leibniz Programmes of the Deutsche Forschungsgemeinschaft and M. Worek by the Initiative and Networking Fund of the Helmholtz Association, contract HA-101 ("Physics at the Terascale").

Note added. Independently of our calculation, another group has evaluated NLO QCD corrections to WWbb production with leptonic decays of gauge bosons, and has presented them in [83]. We have cross checked the results, applying the narrow width approximation for the $\mathrm{W}$ bosons as in that publication, and have obtained perfect agreement for integrated LO and NLO cross sections within statistical errors.

Open Access. This article is distributed under the terms of the Creative Commons Attribution Noncommercial License which permits any noncommercial use, distribution, and reproduction in any medium, provided the original author(s) and source are credited.

\section{References}

[1] P. Nason, S. Dawson and R.K. Ellis, The total cross-section for the production of heavy quarks in hadronic collisions, Nucl. Phys. B 303 (1988) 607 [SPIRES].

[2] W. Beenakker, H. Kuijf, W.L. van Neerven and J. Smith, QCD corrections to heavy quark production in pp collisions, Phys. Rev. D 40 (1989) 54 [SPIRES].

[3] P. Nason, S. Dawson and R.K. Ellis, The one particle inclusive differential cross-section for heavy quark production in hadronic collisions, Nucl. Phys. B 327 (1989) 49 [SPIRES].

[4] W. Beenakker, W.L. van Neerven, R. Meng, G.A. Schuler and J. Smith, QCD corrections to heavy quark production in hadron hadron collisions, Nucl. Phys. B 351 (1991) 507 [SPIRES]. 
[5] W. Bernreuther, A. Brandenburg, Z.G. Si and P. Uwer, Top quark spin correlations at hadron colliders: predictions at next-to-leading order QCD, Phys. Rev. Lett. 87 (2001) 242002 [hep-ph/0107086] [SPIRES].

[6] W. Bernreuther, A. Brandenburg, Z.G. Si and P. Uwer, Top quark pair production and decay at hadron colliders, Nucl. Phys. B 690 (2004) 81 [hep-ph/0403035] [SPIRES].

[7] W. Bernreuther and Z.-G. Si, Distributions and correlations for top quark pair production and decay at the Tevatron and LHC, Nucl. Phys. B 837 (2010) 90 [arXiv:1003.3926] [SPIRES].

[8] K. Melnikov and M. Schulze, NLO QCD corrections to top quark pair production and decay at hadron colliders, JHEP 08 (2009) 049 [arXiv: 0907.3090] [SPIRES].

[9] M. Czakon and A. Mitov, Inclusive heavy flavor hadroproduction in NLO QCD: the exact analytic result, Nucl. Phys. B 824 (2010) 111 [arXiv:0811.4119] [SPIRES].

[10] M. Czakon, Tops from light quarks: full mass dependence at two-loops in QCD, Phys. Lett. B 664 (2008) 307 [arXiv:0803.1400] [SPIRES].

[11] R. Bonciani, A. Ferroglia, T. Gehrmann, D. Maître and C. Studerus, Two-loop fermionic corrections to heavy-quark pair production: the quark-antiquark channel, JHEP 07 (2008) 129 [arXiv:0806.2301] [SPIRES].

[12] C. Anastasiou and S.M. Aybat, The one-loop gluon amplitude for heavy-quark production at NNLO, Phys. Rev. D 78 (2008) 114006 [arXiv:0809.1355] [SPIRES].

[13] B. Kniehl, Z. Merebashvili, J.G. Korner and M. Rogal, Heavy quark pair production in gluon fusion at next-to-next-to-leading $O\left(\alpha_{s}^{4}\right)$ order: one-loop, Phys. Rev. D 78 (2008) 094013 [arXiv:0809.3980] [SPIRES].

[14] R. Bonciani, A. Ferroglia, T. Gehrmann and C. Studerus, Two-loop planar corrections to heavy-quark pair production in the quark-antiquark channel, JHEP 08 (2009) 067 [arXiv:0906.3671] [SPIRES].

[15] R. Bonciani, A. Ferroglia, T. Gehrmann, A. Manteuffel and C. Studerus, Two-loop leading color corrections to heavy-quark pair production in the gluon fusion channel, JHEP 01 (2011) 102 [arXiv:1011.6661] [SPIRES].

[16] M. Czakon and A. Mitov, On the soft-gluon resummation in top quark pair production at hadron colliders, Phys. Lett. B $6 \mathbf{6 0}$ (2009) 154 [arXiv:0812.0353] [SPIRES].

[17] N. Kidonakis, Two-loop soft anomalous dimensions and NNLL resummation for heavy quark production, Phys. Rev. Lett. 102 (2009) 232003 [arXiv:0903.2561] [SPIRES].

[18] M. Beneke, P. Falgari and C. Schwinn, Soft radiation in heavy-particle pair production: all-order colour structure and two-loop anomalous dimension, Nucl. Phys. B $\mathbf{8 2 8}$ (2010) 69 [arXiv:0907.1443] [SPIRES].

[19] M. Czakon, A. Mitov and G.F. Sterman, Threshold resummation for top-pair hadroproduction to next-to-next-to-leading log, Phys. Rev. D 80 (2009) 074017 [arXiv:0907.1790] [SPIRES].

[20] M. Beneke, M. Czakon, P. Falgari, A. Mitov and C. Schwinn, Threshold expansion of the $g g(q q) \rightarrow Q Q+X$ cross section at $O\left(\alpha_{s}^{4}\right)$, Phys. Lett. B $690(2010) 483$ [arXiv:0911.5166] [SPIRES].

[21] V. Ahrens, A. Ferroglia, M. Neubert, B.D. Pecjak and L.L. Yang, Renormalization-group improved predictions for top-quark pair production at hadron colliders, JHEP 09 (2010) 097 [arXiv: 1003.5827] [SPIRES]. 
[22] N. Kidonakis, Next-to-next-to-leading soft-gluon corrections for the top quark cross section and transverse momentum distribution, Phys. Rev. D 82 (2010) 114030 [arXiv:1009.4935] [SPIRES].

[23] W. Beenakker et al., Higgs radiation off top quarks at the Tevatron and the LHC, Phys. Rev. Lett. 87 (2001) 201805 [hep-ph/0107081] [SPIRES].

[24] L. Reina, S. Dawson and D. Wackeroth, QCD corrections to associated tt̄h production at the Tevatron, Phys. Rev. D 65 (2002) 053017 [hep-ph/0109066] [SPIRES].

[25] L. Reina and S. Dawson, Next-to-leading order results for tith production at the Tevatron, Phys. Rev. Lett. 87 (2001) 201804 [hep-ph/0107101] [SPIRES].

[26] W. Beenakker et al., NLO QCD corrections to $t \bar{t} H$ production in hadron collisions, Nucl. Phys. B 653 (2003) 151 [hep-ph/0211352] [SPIRES].

[27] S. Dawson, L.H. Orr, L. Reina and D. Wackeroth, Associated top quark Higgs boson production at the LHC, Phys. Rev. D 67 (2003) 071503 [hep-ph/0211438] [SPIRES].

[28] S. Dawson, C. Jackson, L.H. Orr, L. Reina and D. Wackeroth, Associated Higgs production with top quarks at the Large Hadron Collider: NLO QCD corrections, Phys. Rev. D 68 (2003) 034022 [hep-ph/0305087] [SPIRES].

[29] SM and NlO Multileg Working Group collaboration, J.R. Andersen et al., The SM and NLO multileg working group: summary report, arXiv:1003.1241 [SPIRES].

[30] S. Dittmaier, P. Uwer and S. Weinzierl, NLO QCD corrections to $t \bar{t}+$ jet production at hadron colliders, Phys. Rev. Lett. 98 (2007) 262002 [hep-ph/0703120] [SPIRES].

[31] S. Dittmaier, P. Uwer and S. Weinzierl, Hadronic top-quark pair production in association with a hard jet at next-to-leading order QCD: Phenomenological studies for the Tevatron and the LHC, Eur. Phys. J. C 59 (2009) 625 [arXiv:0810.0452] [SPIRES].

[32] K. Melnikov and M. Schulze, NLO QCD corrections to top quark pair production in association with one hard jet at hadron colliders, Nucl. Phys. B 840 (2010) 129 [arXiv: 1004.3284] [SPIRES].

[33] A. Lazopoulos, T. McElmurry, K. Melnikov and F. Petriello, Next-to-leading order QCD corrections to $t \bar{t} Z$ production at the LHC, Phys. Lett. B 666 (2008) 62 [arXiv:0804.2220] [SPIRES].

[34] D. Peng-Fei et al., QCD corrections to associated production of $t \bar{t} \gamma$ at hadron colliders, arXiv:0907.1324 [SPIRES].

[35] A. Bredenstein, A. Denner, S. Dittmaier and S. Pozzorini, NLO QCD corrections to top anti-top bottom anti-bottom production at the LHC: 1. Quark-antiquark annihilation, JHEP 08 (2008) 108 [arXiv:0807.1248] [SPIRES].

[36] A. Bredenstein, A. Denner, S. Dittmaier and S. Pozzorini, NLO QCD corrections to $p p \rightarrow t \bar{t} b \bar{b}+X$ at the LHC, Phys. Rev. Lett. 103 (2009) 012002 [arXiv:0905.0110] [SPIRES].

[37] G. Bevilacqua, M. Czakon, C.G. Papadopoulos, R. Pittau and M. Worek, Assault on the NLO Wishlist: $p p \rightarrow t t b b$, JHEP 09 (2009) 109 [arXiv: 0907.4723] [SPIRES].

[38] A. Bredenstein, A. Denner, S. Dittmaier and S. Pozzorini, NLO QCD corrections to top anti-top bottom anti-bottom production at the LHC: 2. Full hadronic results, JHEP 03 (2010) 021 [arXiv: 1001.4006] [SPIRES]. 
[39] G. Bevilacqua, M. Czakon, C.G. Papadopoulos and M. Worek, Dominant QCD backgrounds in Higgs boson analyses at the LHC: a study of $p p \rightarrow t \bar{t}+2$ jets at next-to-leading order, Phys. Rev. Lett. 104 (2010) 162002 [arXiv:1002.4009] [SPIRES].

[40] A. Denner, S. Dittmaier, M. Roth and D. Wackeroth, Predictions for all processes $e^{+} e^{-} \rightarrow 4$ fermions $+\gamma$, Nucl. Phys. B 560 (1999) 33 [hep-ph/9904472] [SPIRES].

[41] A. Denner, S. Dittmaier, M. Roth and L.H. Wieders, Electroweak corrections to charged-current $e^{+} e^{-} \rightarrow 4$ fermion processes: technical details and further results, Nucl. Phys. B 724 (2005) 247 [hep-ph/0505042] [SPIRES].

[42] M. Czakon, C.G. Papadopoulos and M. Worek, Polarizing the dipoles, JHEP 08 (2009) 085 [arXiv: 0905.0883] [SPIRES].

[43] A. Kanaki and C.G. Papadopoulos, HELAC: a package to compute electroweak helicity amplitudes, Comput. Phys. Commun. 132 (2000) 306 [hep-ph/0002082] [SPIRES].

[44] A. Cafarella, C.G. Papadopoulos and M. Worek, Helac-Phegas: a generator for all parton level processes, Comput. Phys. Commun. 180 (2009) 1941 [arXiv:0710.2427] [SPIRES].

[45] T. Gleisberg, F. Krauss, C.G. Papadopoulos, A. Schaelicke and S. Schumann, Cross sections for multi-particle final states at a linear collider, Eur. Phys. J. C 34 (2004) 173 [hep-ph/0311273] [SPIRES].

[46] C.G. Papadopoulos and M. Worek, Multi-parton cross sections at hadron colliders, Eur. Phys. J. C 50 (2007) 843 [hep-ph/0512150] [SPIRES].

[47] J. Alwall et al., Comparative study of various algorithms for the merging of parton showers and matrix elements in hadronic collisions, Eur. Phys. J. C 53 (2008) 473 [arXiv:0706.2569] [SPIRES].

[48] C. Englert, B. Jager, M. Worek and D. Zeppenfeld, Observing strongly interacting vector boson systems at the CERN Large Hadron Collider, Phys. Rev. D 80 (2009) 035027 [arXiv:0810.4861] [SPIRES].

[49] S. Actis et al., Quest for precision in hadronic cross sections at low energy: Monte Carlo tools vs. experimental data, Eur. Phys. J. C 66 (2010) 585 [arXiv:0912.0749] [SPIRES].

[50] A. van Hameren, PARNI for importance sampling and density estimation, Acta Phys. Polon. B 40 (2009) 259 [arXiv:0710.2448] [SPIRES].

[51] A. van Hameren, Kaleu: a general-purpose parton-level phase space generator, arXiv: 1003.4953 [SPIRES].

[52] C.G. Papadopoulos, PHEGAS: a phase space generator for automatic cross-section computation, Comput. Phys. Commun. 137 (2001) 247 [hep-ph/0007335] [SPIRES].

[53] MCFM - Monte Carlo for FeMtobarn processes, http://mcfm.fnal.gov/.

[54] A. van Hameren, C.G. Papadopoulos and R. Pittau, Automated one-loop calculations: a proof of concept, JHEP 09 (2009) 106 [arXiv: 0903.4665] [SPIRES].

[55] G. Ossola, C.G. Papadopoulos and R. Pittau, Reducing full one-loop amplitudes to scalar integrals at the integrand level, Nucl. Phys. B 763 (2007) 147 [hep-ph/0609007] [SPIRES].

[56] G. Ossola, C.G. Papadopoulos and R. Pittau, CutTools: a program implementing the OPP reduction method to compute one-loop amplitudes, JHEP 03 (2008) 042 [arXiv:0711.3596] [SPIRES]. 
[57] P. Draggiotis, M.V. Garzelli, C.G. Papadopoulos and R. Pittau, Feynman rules for the rational part of the QCD 1-loop amplitudes, JHEP 04 (2009) 072 [arXiv:0903.0356] [SPIRES].

[58] A. van Hameren, OneLOop: for the evaluation of one-loop scalar functions, arXiv: 1007.4716 [SPIRES].

[59] W. Beenakker et al., The Fermion loop scheme for finite width effects in $e^{+} e^{-}$annihilation into four fermions, Nucl. Phys. B 500 (1997) 255 [hep-ph/9612260] [SPIRES].

[60] E.N. Argyres et al., Stable calculations for unstable particles: Restoring gauge invariance, Phys. Lett. B 358 (1995) 339 [hep-ph/9507216] [SPIRES].

[61] S. Dittmaier, Separation of soft and collinear singularities from one- loop N-point integrals, Nucl. Phys. B 675 (2003) 447 [hep-ph/0308246] [SPIRES].

[62] S. Catani and M.H. Seymour, A general algorithm for calculating jet cross sections in NLO QCD, Nucl. Phys. B 485 (1997) 291 [hep-ph/9605323] [SPIRES].

[63] S. Catani, S. Dittmaier, M.H. Seymour and Z. Trócsányi, The dipole formalism for next-to-leading order QCD calculations with massive partons, Nucl. Phys. B 627 (2002) 189 [hep-ph/0201036] [SPIRES].

[64] R. Kleiss and R. Pittau, Weight optimization in multichannel Monte Carlo, Comput. Phys. Commun. 83 (1994) 141 [hep-ph/9405257] [SPIRES].

[65] M. Dinsdale, M. Ternick and S. Weinzierl, Parton showers from the dipole formalism, Phys. Rev. D 76 (2007) 094003 [arXiv:0709.1026] [SPIRES].

[66] S. Schumann and F. Krauss, A parton shower algorithm based on Catani-Seymour dipole factorisation, JHEP 03 (2008) 038 [arXiv:0709.1027] [SPIRES].

[67] Particle Data Group collaboration, C. Amsler et al., Review of particle physics, Phys. Lett. B 667 (2008) 1 [SPIRES].

[68] M. Jezabek and J.H. Kuhn, QCD corrections to semileptonic decays of heavy quarks, Nucl. Phys. B 314 (1989) 1 [SPIRES].

[69] K.G. Chetyrkin, R. Harlander, T. Seidensticker and M. Steinhauser, Second order QCD corrections to $\Gamma(t \rightarrow W b)$, Phys. Rev. D 60 (1999) 114015 [hep-ph/9906273] [SPIRES].

[70] S. Catani, Y.L. Dokshitzer and B.R. Webber, The $K^{-}$perpendicular clustering algorithm for jets in deep inelastic scattering and hadron collisions, Phys. Lett. B 285 (1992) 291 [SPIRES].

[71] S. Catani, Y.L. Dokshitzer, M.H. Seymour and B.R. Webber, Longitudinally invariant $K_{t}$ clustering algorithms for hadron hadron collisions, Nucl. Phys. B 406 (1993) 187 [SPIRES].

[72] S.D. Ellis and D.E. Soper, Successive combination jet algorithm for hadron collisions, Phys. Rev. D 48 (1993) 3160 [hep-ph/9305266] [SPIRES].

[73] M. Cacciari, G.P. Salam and G. Soyez, The anti-k $k_{t}$ jet clustering algorithm, JHEP 04 (2008) 063 [arXiv:0802.1189] [SPIRES].

[74] Y.L. Dokshitzer, G.D. Leder, S. Moretti and B.R. Webber, Better jet clustering algorithms, JHEP 08 (1997) 001 [hep-ph/9707323] [SPIRES].

[75] J. Pumplin et al., New generation of parton distributions with uncertainties from global QCD analysis, JHEP 07 (2002) 012 [hep-ph/0201195] [SPIRES]. 
[76] D. Stump et al., Inclusive jet production, parton distributions and the search for new physics, JHEP 10 (2003) 046 [hep-ph/0303013] [SPIRES].

[77] F. Halzen, P. Hoyer and C.S. Kim, Forward-backward asymmetry of hadroproduced heavy quarks in QCD, Phys. Lett. B 195 (1987) 74 [SPIRES].

[78] J.H. Kuhn and G. Rodrigo, Charge asymmetry in hadroproduction of heavy quarks, Phys. Rev. Lett. 81 (1998) 49 [hep-ph/9802268] [SPIRES].

[79] J.H. Kuhn and G. Rodrigo, Charge asymmetry of heavy quarks at hadron colliders, Phys. Rev. D 59 (1999) 054017 [hep-ph/9807420] [SPIRES].

[80] O. Antunano, J.H. Kuhn and G. Rodrigo, Top quarks, axigluons and charge asymmetries at hadron colliders, Phys. Rev. D 77 (2008) 014003 [arXiv:0709.1652] [SPIRES].

[81] CDF collaboration, T. Aaltonen et al., Measurement of the inclusive forward-backward asymmetry and its rapidity dependence $A_{F B}(\Delta y)$ in $t \bar{t}$ production in $5.3 \mathrm{fb}^{-1}$ of TeVatron data, CDF-CONF-NOTE-10185 (2010).

[82] DØ collaboration, V.M. Abazov et al., Measurement of the forward-backward production asymmetry of $t$ and $\bar{t}$ quarks in $p \bar{p} \rightarrow t \bar{t}$ events, D0-CONF-NOTE-6062 (2010).

[83] A. Denner, S. Dittmaier, S. Kallweit and S. Pozzorini, NLO QCD corrections to WWbb production at hadron colliders, arXiv:1012.3975 [SPIRES]. 